\title{
Impact of different intensities of intermittent theta burst stimulation on the cortical properties during TMS-EEG and working memory performance
}

\author{
Sung Wook Chung ${ }^{1}$ (1) | Nigel C. Rogasch ${ }^{2}$ | Kate E. Hoy ${ }^{1}$ | Caley M. Sullivan ${ }^{1}$ | \\ Robin F. H. Cash ${ }^{1}$ | Paul B. Fitzgerald ${ }^{1,3}$
}

${ }^{1}$ Monash Alfred Psychiatry Research Centre, Monash University, Central Clinical School and The Alfred, Melbourne, Australia

${ }^{2}$ Brain and Mental Health Laboratory, School of Psychological Sciences and Monash Biomedical Imaging, Monash Institute of Cognitive and Clinical Neuroscience, Monash University, Melbourne, Australia

${ }^{3}$ Epworth Clinic, Epworth Healthcare, Camberwell, VIC, Australia

\section{Correspondence}

Sung Wook Chung, Monash Alfred Psychiatry Research Centre, Level 4, 607 St Kilda Road, Melbourne, Victoria, Australia, 3004.

Email: sung.chung@monash.edu

\section{Funding information}

SWC was supported by a Monash

Graduate Scholarship. NCR is supported by a NHMRC Early Career Fellowship (1072057). KEH is supported by a NHMRC Career Development Fellowship (1082894). PBF is supported by a NHMRC Practitioner Fellowship (1078567). PBF has received equipment for research from MagVenture A/S, Medtronic Ltd, Cervel Neurotech and Brainsway Ltd and funding for research from Neuronetics and Cervel Neurotech. $\mathrm{He}$ is on the scientific advisory board for Bionomics Ltd.

\begin{abstract}
Intermittent theta burst stimulation (iTBS) is a noninvasive brain stimulation technique capable of increasing cortical excitability beyond the stimulation period. Due to the rapid induction of modulatory effects, prefrontal application of iTBS is gaining popularity as a therapeutic tool for psychiatric disorders such as depression. In an attempt to increase efficacy, higher than conventional intensities are currently being applied. The assumption that this increases neuromodulatory may be mechanistically false for iTBS. This study examined the influence of intensity on the neurophysiological and behavioural effects of TTBS in the prefrontal cortex. Sixteen healthy participants received iTBS over prefrontal cortex at either 50,75 or $100 \%$ resting motor threshold in separate sessions. Single-pulse TMS and concurrent electroencephalography (EEG) was used to assess changes in cortical reactivity measured as TMS-evoked potentials and oscillations. The $n$-back task was used to assess changes in working memory performance. The data can be summarised as an inverse U-shape relationship between intensity and iTBS plastic effects, where 75\% iTBS yielded the largest neurophysiological changes. Improvement in reaction time in the 3-back task was supported by the change in alpha power, however, comparison between conditions revealed no significant differences. The assumption that higher intensity results in greater neuromodulatory effects may be false, at least in healthy individuals, and should be carefully considered for clinical populations. Neurophysiological changes associated with working memory following iTBS suggest functional relevance. However, the effects of different intensities on behavioural performance remain elusive in the present healthy sample.
\end{abstract}

\section{KEYWORDS}

intensity, prefrontal cortex, theta burst stimulation (TBS), TMS-EEG, working memory

\section{1 | INTRODUCTION}

Repetitive transcranial magnetic stimulation (rTMS) is a noninvasive brain stimulation technique capable of modulating cortical activity beyond the stimulation period. Clinical applications of rTMS have been studied in various neurological and psychiatric disorders (Machado et al., 2013), especially in the treatment of depression (George et al., 2010; George, Taylor, \& Short, 2013; O'Reardon et al., 2007). Recently, a modified form of rTMS known as theta-burst stimulation (TBS), has been investigated as a potential treatment of depression, with promising therapeutic effects (Chung, Hoy, \& Fitzgerald, 2015a; Duprat et al., 2016; Li et al., 2014). TBS was modified from an animal stimulation paradigm (Larson, Wong, \& Lynch, 1986) and elicits long-term potentiation or depression (LTP/LTD)-like changes depending on the stimulation pattern in human (Huang, Edwards, Rounis, Bhatia, \& Rothwell, 2005). In the motor cortex, intermittent TBS (iTBS, $2 \mathrm{sec}$ on, $8 \mathrm{sec}$ off, 600 pulses, 3 minutes duration) elicits LTP-like increases in cortical excitability whereas continuous TBS (cTBS, $40 \mathrm{sec}$ on, 600 pulses) evokes LTD-like decreases cortical excitability (Huang et al., 2005; Suppa et al., 2008). Due to the rapid induction of modulatory effects 
compared to conventional rTMS, TBS is an attractive option for neuromodulatory treatments in clinical disorders (Chung et al., 2015a; Machado et al., 2013). For psychiatric conditions, this typically involves stimulation delivered to prefrontal cortical regions such as dorsolateral prefrontal cortex (DLPFC) (Desmyter et al., 2016; Li et al., 2014; Plewnia et al., 2014). As adoption of TBS increases in the clinical literature, the lack of consensus with respect to optimal intensity is becoming increasingly evident. Conventionally, TBS in the motor cortex has been applied at $80 \%$ of active motor threshold (aMT) (Huang et al., 2005), equivalent to approximately $70 \%$ of resting motor threshold (rMT) (Cardenas-Morales et al., 2014; Chen et al., 1998; Gentner, Wankerl, Reinsberger, Zeller, \& Classen, 2008). Recent reports of the stimulation intensity used in prefrontal TBS for therapeutic intervention have varied quite substantially in the range of $80-120 \%$ of rMT (Bakker et al., 2015; Desmyter et al., 2016; Duprat et al., 2016; Li et al., 2014; Plewnia et al., 2014; Prasser et al., 2015). The underlying assumption is that the efficacy of iTBS will be greater with increasing intensity of stimulation. This is partially supported by linear responses to increases in the intensity of conventional rTMS in healthy individuals $(1 \mathrm{~Hz}$ [Nahas et al., 2001]) and in clinical populations (10 Hz [Padberg et al., 2002]). Studies using different modulatory paradigms have also shown a shift from LTD- to LTP-like effects at higher intensity (Batsikadze, Moliadze, Paulus, Kuo, \& Nitsche, 2013; Cash, Jegatheeswaran, Ni, \& Chen, 2017a; Doeltgen \& Ridding, 2011), corroborating the idea of increased propensity for LTP-like changes at higher intensity. However, systematic investigation of intensity-dependent effects of iTBS in the prefrontal cortex has not been established.

Another key question concerns the use of TBS for cognitive disorders. TBS was originally developed to mimic the natural firing patterns of neurons in the hippocampus, where high-frequency gamma oscillations $(30-80 \mathrm{~Hz})$ were modulated by the phase of lower frequency theta oscillations (4-7 Hz) (Lisman \& Jensen, 2013). Applying electrical stimulation to the hippocampus with gamma frequency bursts nested in theta frequency rhythms resulted in robust long-term potentiation (LTP) (Larson et al., 1986). A similar theta-gamma coupling relationship in endogenous brain activity has been observed in human studies using electroencephalography (EEG) during cognitive functions (Lisman, 2010). It is therefore of particular interest whether iTBS in human can facilitate cognitive and memory processes, and to what extent the plastic changes elicited by TBS translate to changes in neurophysiological metrics of cognition and behavioural performance outcomes.

Recent advances in technology have enabled the measurement of plastic neuronal changes following neuromodulatory paradigms using concurrent recording of electroencephalographic responses to TMS (TMS-EEG) (Chung, Rogasch, Hoy, \& Fitzgerald, 2015b; Farzan et al., 2016; Hill, Rogasch, Fitzgerald, \& Hoy, 2016). Each TMS pulse elicits a TMS-evoked EEG response, and the change in the amplitude of TMSevoked potentials (TEPS) and the power of TMS-evoked oscillations following TBS provide metric of plasticity in the prefrontal cortex (Chung et al., 2017). TEPs are composed of several components which are thought to represent excitatory and inhibitory postsynaptic potentials. A negative trough at a latency of approximately $100 \mathrm{~ms}$ (N100) has been associated with inhibitory mechanisms in motor (Bonnard,
Spieser, Meziane, de Graaf, \& Pailhous, 2009; Premoli et al., 2014; Rogasch, Daskalakis, \& Fitzgerald, 2013a) and prefrontal cortex (Chung et al., 2017; Rogasch, Daskalakis, \& Fitzgerald, 2015), and is considered to be the most robust TEP component with the best signal to noise ratio (SNR) (Noda et al., 2016). Modulation of this component has been observed following TBS over the prefrontal cortex (Chung et al., 2017) and cerebellum (Casula et al., 2016b; Harrington \& Hammond-Tooke, 2015). Recent studies also suggest that a peak at a latency of $60 \mathrm{~ms}$ (P60) may be a correlate of neuronal excitability in motor cortex (Cash et al., 2017b) and DLPFC (Hill, Rogasch, Fitzgerald, \& Hoy, 2017).

In the present study, we examined the relationship between iTBS intensity (50, 75 and $100 \%$ of individual rMT), LTP-like neural plasticity and the relationship to neurophysiological and behavioural metrics of learning and memory using N-back task (Haatveit et al., 2010). We hypothesized that iTBS would be accompanied by plastic changes in N100 and P60 amplitude. Secondly, we anticipated that the efficacy of iTBS would increase with increasing intensity. Thirdly, we hypothesized that these changes will be mirrored by increasing working memory (WM) performance measured via N-back task and neurophysiological correlates. The modulation of theta and gamma oscillatory activity was of particular interest since these frequency bands are targeted by TBS and involved in WM.

\section{2 | MATERIAL AND METHODS}

\section{1 | Participants}

Sixteen healthy volunteers ( 7 female, $27.8 \pm 8.6$ years of age, $16.25 \pm$ 2.11 years of formal education) participated in the study. All subjects were right-handed according to the Edinburgh Handedness Inventory, and the mini international neuropsychiatric interview (MINI) was performed (Sheehan et al., 1998) to confirm no history of mental illness. No participants were smokers. All participants provided informed consent prior to the experiment and the experimental procedures were approved by the Alfred Hospital and Monash University Human Research Ethics Committees.

\section{2 | Procedure}

Each participant attended 3 sessions receiving iTBS at either 50\%, 75\% or $100 \%$ of their resting motor threshold (rMT). Each session was at least 72 hours apart and the session order was pseudorandomized across participants. The experimental procedures comprised of recording EEG during 50 single TMS pulses before ( $\mathrm{BL}$-baseline), 5 min post (T5) and 30 min post (T30) iTBS (Figure 1a). The N-back WM task (2back and 3-back conditions) was also performed pre (BL) and 15 min post (T15) iTBS with concurrent EEG recording.

\section{3 | EEG recording}

EEG was recorded with $\mathrm{TMS}$-compatible $\mathrm{Ag} / \mathrm{AgCl}$ electrodes and a DC-coupled amplifier (SynAmps2, EDIT Compumedics Neuroscan, Texas, USA). 42 electrodes were used on a 64-channel EEG cap (AF3, AF4, F7, F5, F3, F1, Fz, F2, F4, F6, F8, FC5, FC3, FC1, FCz, FC2, FC4, 


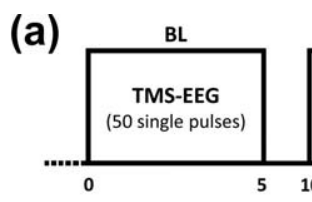

(b)

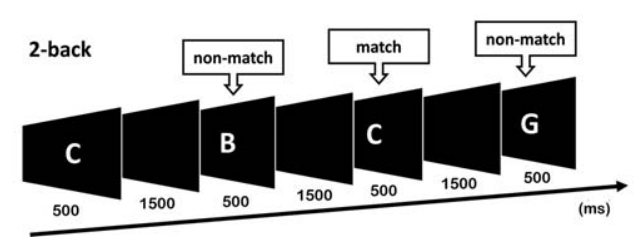

(c)

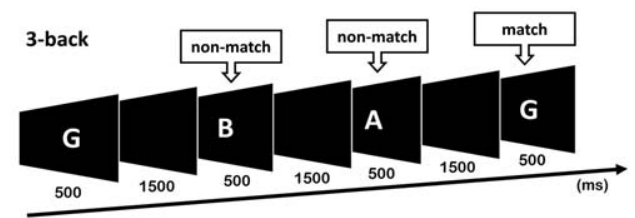

FIGURE 1 Schematic diagram of the experimental design. (a) Concurrent recording of electroencephalogram during transcranial magnetic stimulation (TMS-EEG) and N-back task were performed at baseline (BL). Intermittent theta burst stimulation (iTBS) was then administered at one of three intensities. TMS-EEG was rerepeated at T5 and T30 following iTBS, and the N-back at T15 following iTBS. (b-c) Diagrams illustrating trials of match and nonmatch during 2-back and 3-back tasks

FC6, T7, C5, C3, C1, Cz, C2, C4, C6, T8, P7, P5, P3, P1, Pz, P2, P4, P6, $\mathrm{P} 8, \mathrm{PO} 3, \mathrm{PO}, \mathrm{PO} 4, \mathrm{O} 1, \mathrm{Oz}, \mathrm{O} 2)$, and electrooculography recording was obtained with 4 electrodes, one positioned above and one below the left eye and one on lateral to the outer canthus of either eye. Electrodes were online referenced to $\mathrm{CPz}$ and grounded to FPz with exception to the lateral eye electrodes which were referenced to each other. For TMS-EEG, data were recorded with a high acquisition rate $(10,000$ $\mathrm{Hz}$ ) using a large operating range $( \pm 200 \mathrm{mV}$ ) to avoid amplifier saturation. Signals were amplified $(1,000 \times)$ and low pass filtered (DC-2,000 $\mathrm{Hz}$ ). For EEG recording during $\mathrm{N}$-back task, $\mathrm{AC}$ acquisition setting was used and the signals were filtered (low pass at $200 \mathrm{~Hz}$, high pass at $0.05 \mathrm{~Hz}$ ) and sampled at $1,000 \mathrm{~Hz}$ with an operating window of \pm 950 $\mu \mathrm{V}$. Electrode impedance levels were kept below $5 \mathrm{k} \Omega$ throughout the experiment. During TMS-EEG recording, subjects listened to white noise through intra-auricular earphones (Etymotic Research, ER3-14A, USA) to limit the influence of the auditory processing of the TMS click. The sound level was adjusted for each individual subject until singlepulse TMS at $120 \%$ rMT was barely audible.

\section{4 | Transcranial magnetic stimulation}

Participants sat comfortably with their arms resting on a pillow throughout the experiment. The EEG cap was mounted following the 10-20 standard system and the resting motor threshold (rMT) was obtained from left motor cortex, which was identified as the minimum intensity required to evoke at least 3 out of 6 motor evoked potentials (MEPs) $>0.05 \mathrm{mV}$ in amplitude (Conforto, Z'graggen, Kohl, Rosler, \& Kaelin-Lang, 2004) via $\mathrm{Ag} / \mathrm{AgCl}$ electromyography electrodes attached to the first dorsal interosseous (FDI) muscles. TMS was administered to the left prefrontal cortex at F1 electrode using 10/20 method of placement. The F1 electrode sits over the superior frontal gyrus with Brodmann area (BA) of 6, 8, and 9 (Koessler et al., 2009), and therefore, is part of dorsolateral prefrontal cortex. This electrode was chosen to minimize stimulation of scalp muscles which result in large artefacts in EEG recordings lasting up to $40 \mathrm{~ms}$ following the TMS pulse (Rogasch, Thomson, Daskalakis, \& Fitzgerald, 2013b). By minimising artefacts, the amount of correction needed in postprocessing for the TMS-EEG signals is reduced. A MagVenture B-65 fluid-cooled coil (a figure-ofeight coil; MagVenture A/S, Denmark) was used for both single-pulse stimulation and iTBS (biphasic pulses, antero-posterior to posteroanterior current direction in the underlying cortex). The coil was positioned at $45^{\circ}$ angle relative to midline, which has been shown to produce strongest stimulation in the prefrontal cortex (Thomson et al., 2013). A line was drawn on the coil at $45^{\circ}$ angle, which would then sit perpendicular to the midline of the EEG cap to ensure same angle positioning. In addition, the edge of the coil was marked on the cap to reliably re-position the coil within $5 \mathrm{~mm}$ (Rogasch et al., 2013b).

Participants received 50 single pulses to left prefrontal cortex at an interval of $5 \mathrm{~s}$ (10\% jitter) at 120\% rMT before and after different stimulation intensities of iTBS. These parameters were chosen to be consistent with our previous study (Chung et al., 2017), and the majority of the TMS-EEG studies in the prefrontal cortex used suprathreshold intensities (Cash et al., 2017b; Daskalakis et al., 2008; Farzan et al., 2009, 2010; Hill et al., 2017; Kahkonen, Wilenius, Nikulin, Ollikainen, \& Ilmoniemi, 2003; Rogasch et al., 2014) which may also allowed for better signal-to-noise ratio, given that smaller TEPs are obtained in the prefrontal cortex compared to the motor cortex (Kahkonen, Komssi, Wilenius, \& Ilmoniemi, 2005). Across different sessions, participants received iTBS at different intensities $(50 \%, 75 \%$, or $100 \%$ rMT). 75\% rMT was chosen as smaller EEG responses to TMS have been observed in the prefrontal cortex compared to the motor cortex (Kahkonen et al., 2005) and to be within the range of $70-80 \%$ motor threshold as previously been described (Gentner et al., 2008; Goldsworthy, Pitcher, \& Ridding, 2012; Jones et al., 2016; Nettekoven et al., 2014; Pedapati et al., 2015, 2016; Tsang et al., 2014). In addition, the intensity was set relative to $\mathrm{rMT}$ rather than aMT to avoid potential metaplastic influences related to prior muscle activation (Cash, Mastaglia, \& Thickbroom, 2013). With the exception of intensity, iTBS parameters adhered to the originally described method (Huang et al., 2005). iTBS consisted of a burst of 3 pulses given at $50 \mathrm{~Hz}$ repeated at a frequency of $5 \mathrm{~Hz}$, with $2 \mathrm{~s}$ of stimulation on and $8 \mathrm{~s}$ off repeated for a total of 600 pulses. The average stimulation intensity was as follows (\% of maximum stimulator output; mean \pm SD): $50 \%$ condition $=28.5 \pm$ $3.0 \% ; 75 \%$ condition $=42.5 \pm 5.3 \% ; 100 \%$ condition $=57.19 \pm 5.6 \%$. 


\section{5 | Working memory task}

Participants were assessed on the N-back task with 5 mins of 2-back and 5 mins of 3-back conditions in a pseudorandomised order. Letters were in a random series of $A$ to $J$, and participants were requested to respond with a button press when the presented letter was the same as the letter appeared either 2 trials (Figure 1b; 2-back) or 3 trials (Figure 1c; 3-back) earlier. Each letter was presented in white on a black screen for 500 ms with a 1,500 ms interstimulus interval. Each N-back task consisted of 130 trials with $25 \%$ targets. Due to technical failure, data was not collected from one participant (complete data from 15 participants; $27.3 \pm 8.7$ years, 7 female). WM performance was assessed via accurate reaction time and $d$ prime sensitivity index $\left(d^{\prime}\right)(z-$ transformed values of hit- minus false-alarm rates) (Haatveit et al., 2010).

\subsection{EEG data preprocessing}

EEG data were analysed offline using EEGLAB (Delorme \& Makeig, 2004), FieldTrip (Oostenveld, Fries, Maris, \& Schoffelen, 2011), TESA (Rogasch et al., 2017) and custom scripts on Matlab platform (R2015b, The MathWorks, USA). For TMS-EEG, data were epoched around the test TMS pulse $(-1,000$ to $1,000 \mathrm{~ms})$, baseline corrected to the TMSfree data $(-500$ to $-50 \mathrm{~ms}$ ), and data around the large signal from TMS pulse $(-5$ to $10 \mathrm{~ms}$ ) were removed and linearly interpolated. The epoched TMS-EEG data from all three time points (BL, T5, T30) were concatenated and analysed concurrently to avoid bias in component rejection. Data were downsampled to $1,000 \mathrm{~Hz}$ and visually inspected to remove epochs with excessive noise (i.e., muscle artefact), and bad channels (i.e., disconnected). An average of 47.6 ( \pm 2.7) trials was included in the 50\% iTBS condition, 47.4 ( \pm 2.8 ) trials in the $75 \%$ iTBS condition and $48.0( \pm 2.7)$ trials in the $100 \%$ iTBS condition across each time point. Two rounds of independent component analysis (FastICA algorithm using the 'tanh' contrast function) were applied to the data; the first to remove large amplitude muscle artefacts, and the second to remove other common artefacts following offline filtering. The first round of independent component analysis (ICA) used a semiautomated component classification algorithm (tesa_compselect function) to remove the remainder of the muscle artefact (Korhonen et al., 2011) (classified if component time course 8 times larger than the mean absolute amplitude across the entire time course). All data were bandpass filtered (second-order, zero-phase, Butterworth filter, 1-80 $\mathrm{Hz}$ ) and bandstop filtered (48-52 Hz; to remove $50 \mathrm{~Hz}$ line noise) and epochs were inspected again to remove any anomalous activity in the EEG trace. The second round of FastICA was conducted, and additional artefactual components were removed based on a previous study (Rogasch et al., 2014) and using TESA toolbox as a guide (Rogasch et al., 2017). Components representing the following artefacts were removed; eye blinks and saccades (mean absolute $z$ score of the two electrodes larger than 2.5), persistent muscle activity (high frequency power that is $60 \%$ of the total power), decay artefacts and other noiserelated artefacts (one or more electrode has an absolute $z$ score of at least 4).
For EEG during N-back tasks, data were epoched around the correctly encoded and maintained trials ( $-1,450$ to $1,990 \mathrm{~ms}$ ), and baseline corrected ( -350 to $-50 \mathrm{~ms}$ ). Trials containing a button response in the epochs were excluded to avoid confounds introduced by motor preparation. Epoched EEG data for two time points (BL, T15) and two Nback tasks were concatenated and analysed concurrently to avoid bias in rejecting components. Data were visually inspected to remove epochs with excessive noise, and bad channels removed. An average of trials included in 50\% iTBS conditions were-76.6 ( \pm 12.9) for 2-back, 74.5 ( \pm 22.2 ) for 3-back; in 75\% iTBS conditions were-74.6 ( \pm 13.3) for 2-back, 78.1 ( \pm 17.8) for 3-back, and in 100\% iTBS conditions were -75.8 ( \pm 14.3) for 2-back, 74.4 ( \pm 24.5 ) for 3-back tasks. It has been demonstrated that late ERP components such as P300 encounters the risk of being distorted following high-pass filter above $1 \mathrm{~Hz}$ (Rousselet, 2012). However, drift in data filtered at $0.1 \mathrm{~Hz}$ is not suitable for ICA (Debener \& De Vos, 2011). Therefore, steps were taken to minimize the distortion of ERPs; (1) All data were bandpass filtered (secondorder, zero-phase, Butterworth filter, $0.1-80 \mathrm{~Hz}$ ) and bandstop filtered (48-52 Hz), and set aside. (2) Original data were bandpass filtered at 1-80 Hz, and FastICA with artefact component removal was conducted as described above (only one round of ICA). (3) The ICA weight matrix from step 2 was then applied to the data in step 1.

For all EEG data, removed channels were interpolated, and data were re-referenced to common average reference. Finally, data were separated into time point blocks (TMS-EEG: BL, T5 and T30; N-back EEG: BL and T15), conditions (50\%, 75\% and 100\% iTBS) and/or tasks (2-back and 3-back).

\section{7 | TMS-evoked potentials (TEPs) and event related potentials (ERPs) during $\mathrm{N}$-back tasks}

TEPs and ERPs were analysed using a global scalp analysis (clusterbased permutation statistics) to access the effect of iTBS across the cortex. For TEPs, the MagVenture stimulator has shown to introduce unwanted artefacts on electrodes in contact with the coil (Rogasch et al., 2013b). As such, the FCz electrode was chosen for TEP waveform representation. Amplitudes of TEPs were compared across time and conditions within predetermined time window for N45 (30-55 ms), P60 (55-80 ms), N100 (90-140 ms) and P200 (160-240 ms). These peaks are known to occur following prefrontal TMS-EEG (Chung et al., 2017; Hill et al., 2017; Rogasch et al., 2014, 2015). A signal-tonoise ratio (SNR) analysis was performed on the average of three fronto-central electrodes (FC1, FCz, FC2) for each individual to validate the limited number of TMS pulses available for the analyses ( $\sim 7$ pulses). The SNR was calculated by dividing the peak amplitude by the standard deviation (SD) of the TEPs in the prestimulus period (-500 to $-50 \mathrm{~ms}$ ) (Chung et al., 2017; Hu, Mouraux, Hu, \& lannetti, 2010).

For ERPs during $\mathrm{N}$-back tasks, the same electrode was used for graphical representation, and peaks were statistically compared within time window for N100 (70-110 ms), P150 (120-180 ms), N200 (190$260 \mathrm{~ms}$ ) and P300 (280-380 ms) during encoding/maintenance period. These peaks were chosen for the implication of these peaks in visual WM tasks (Coull, 1998; Kok, 2001; Vogel \& Luck, 2000). 


\section{8 | TMS-evoked oscillations and event related oscillations during $\mathrm{N}$-back tasks}

TMS-evoked oscillatory power and event related oscillations during $\mathrm{N}$ back tasks were measured by converting TEPs and ERPs into the frequency domain using Morlet wavelet decomposition (3.5 oscillation cycles (Casula et al., 2016b; Hill et al., 2016; Hoy et al., 2016; Rogasch et al., 2015) with steps of $1 \mathrm{~Hz}$ between $2 \mathrm{~Hz}$ and $50 \mathrm{~Hz}, 10 \mathrm{~ms}$ time resolution on each trial for each electrode. The oscillatory power was then averaged to compute the total power of activity, which contained both evoked and induced oscillations. In line with recent discussions on the different approaches to the analysis of oscillatory activity in TMSEEG (Pellicciari, Veniero, \& Miniussi, 2017b), we explored the effects of iTBS on evoked neural oscillations alone. Normalised oscillatory power was then obtained by dividing all power bins by a mean baseline value ( -650 to $-350 \mathrm{~ms}$ ). This baseline window was chosen to avoid the temporal smearing of poststimulus activity into the baseline as the lowest frequency of interest (i.e., $5 \mathrm{~Hz}-200 \mathrm{~ms}$ ) would require at least 350 ms ( 3.5 oscillation cycles $\times 200 \mathrm{~ms}(5 \mathrm{~Hz})=700 \mathrm{~ms}$; Half of the wavelet length $-700 / 2=350 \mathrm{~ms})$. Power values were averaged in frequency bands of interest; theta $(5-7 \mathrm{~Hz})$ and gamma $(30-45 \mathrm{~Hz})$, and in time (50-250 ms for theta, 50-150 ms for gamma) prior to the computation of cluster-based statistics. Focused analyses were conducted on theta and gamma frequencies as theta-burst stimulation is comprised of these two frequency bands, and also due to the implication of synaptic plasticity by the interaction between theta and gamma frequency bands (Zheng \& Zhang, 2015). For the N-back tasks, oscillations were investigated in two blocks; during the letter presentation (50-450 ms) and after the letter presentation (550-950 ms), and averaged across these time windows for both theta and gamma oscillations prior to the cluster-based statistics. Similar to the examination of oscillatory activity during TMS-EEG, evoked oscillations were also investigated.

Additional multi-dimensional cluster-based statistics were performed [time (50-500 ms for TMS-EEG; 50-950 ms for N-back tasks) $\times$ frequency $(5-45 \mathrm{~Hz}) \times$ space], as it is recommended to analyse the data in all possible dimensions (van Ede \& Maris, 2016). Further subgroup analyses on alpha $(8-12 \mathrm{~Hz})$ and beta $(13-29 \mathrm{~Hz})$ bands were also conducted to explore any iTBS-induced change in these frequencies.

\section{9 | Source estimation}

In order to establish the spread of activity following single-pulse TMS on F1 electrode, source estimation was performed. All source localisation was performed using depth-weighted minimum norm estimation (MNE) implemented in Brainstorm software (Tadel, Baillet, Mosher, Pantazis, \& Leahy, 2011) which is documented and freely available for download online under the GNU general public licence (http://neuroimage.usc.edu/brainstorm/). A template anatomy (ICBM 152) in Brainstorm software was used as individual MRI scans were not obtained. The forward model used the Symmetric Boundary Element Method provided by OpenMEEG (Gramfort, Papadopoulo, Olivi, \& Clerc, 2010), and the inverse model was computed with dipole orientations constrained to be normal to the cortex.

\subsection{0 | Addition of control condition}

Twelve age and gender matched participants ( 5 female, $27.8 \pm 7.4$ years of age, $16.5 \pm 2.35$ years of formal education) were included in this study as a control condition for a secondary analysis where no active stimulation was applied. Sham iTBS was applied at $90^{\circ}$ tilt with the bottom of the TMS coil facing away from the scalp. An average of 48.9 ( \pm 1.2) trials were included in the TMS-EEG data, 73.1 ( \pm 18.8) trials in the 2-back, and 72.5 ( \pm 20.6) trials in the 3-back EEG data across each time point.

\subsection{1 | Statistics}

Statistical analysis was performed in SPSS (Version 22) and Matlab. Data did not meet the requirement for normality (Shapiro-Wilk test) in behaviour measures, and therefore nonparametric statistics were used. The Wilcoxon signed-rank tests were conducted for comparison between different pre- and post-iTBS measures to assess whether iTBS conditions altered WM performance. To assess whether iTBS conditions differentially affected WM, Friedman's Analysis of Variance by Ranks was used with a factor of condition (50\%, 75\%, 100\%) to compare the change-from-baseline scores (post-pre; $\Delta$ ) between conditions. For the comparison between active $(n=15)$ and sham $(n=12)$ conditions, the Mann-Whitney $U$ test was performed on the $\Delta$ scores.

For analysis of electrophysiological data, nonparametric clusterbased statics were used (Oostenveld et al., 2011). Monte Carlo p-values were calculated on 5000 random permutations and a value of $p<.05$ was used as the cluster-statistical significance for all analyses, controlling for multiple comparisons across space and time $(p<.025$; two-tailed test). Within condition comparison was first conducted over time to assess whether iTBS conditions altered peak amplitudes/oscillatory power over time (post-iTBS vs pre-iTBS). To assess whether iTBS conditions differentially modulated these measure, $\Delta$ values (post-pre) were calculated and compared between conditions (dependent $t$-tests between active conditions (within groups), independent $t$ tests between active and sham conditions (between groups)).

To assess the relationship between the changes in TMS-evoked activities, N-back related electrophysiology and WM performances, Spearman's rank correlations were used.

\section{3 | RESULTS}

\section{1 | Single-pulse TMS}

An overview of TEP waveforms following single-pulse TMS over left prefrontal cortex (F1 electrode) and the source estimation at the peaks of interest (N45, P60, N100 and P200) are illustrated in Figure 2. The scalp topography and the source estimation of these peaks conform to other TMS-EEG studies in the prefrontal cortex (Chung et al., 2017; Hill et al., 2017; Rogasch et al., 2014).

The analysis of SNR can be found in the Supporting Information Table S1. Qualitatively N45 peaks showed moderate values ( 2.5 SDs), but other peaks, especially latter peaks (N100 and P200) showed high/ acceptable SNR. 


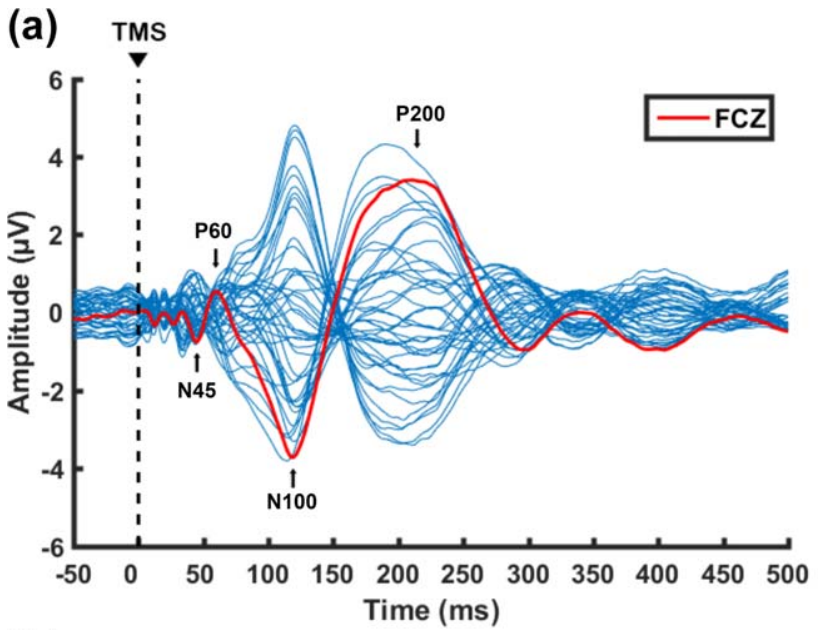

(b)

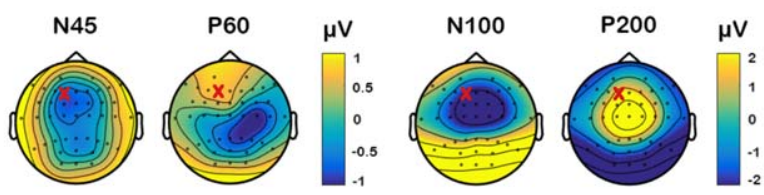

(c)

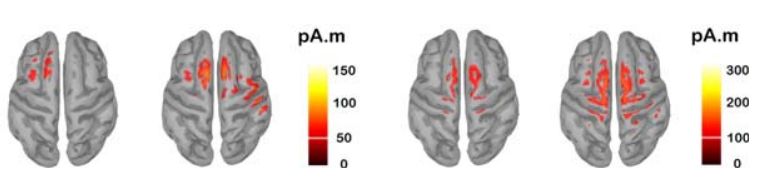

FIGURE 2 Transcranial magnetic stimulation (TMS)-evoked potentials following single-pulse stimulation over left prefrontal cortex (F1 electrode) before theta-burst stimulation (data combined across conditions at baseline). (a) Butterfly plot of all electrodes with peaks of interest (N45, P60, N100, P200) shown in text. The red line indicates the waveform obtained from $\mathrm{FCz}$ electrode for graphical representation. (b) Voltage distribution and (c) Minimum Norm Estimates (MNEs) of the source level activity at the cortex for each peak of interest. ' $\mathrm{X}$ ' on topoplots represents stimulation site

\subsection{The effect of different iTBS intensities on TMS- evoked activity}

We first assessed the after-effects of iTBS by comparing the amplitudes of TEPs over time, and across conditions. Using the clusterbased permutation tests between pre-iTBS (BL) and 5-min post iTBS (T5), we found that both 50\% (115-140 ms, $p=.011$, right frontal; Figure $3 a)$ and $75 \%$ iTBS (110-140 ms, $p=.010$, bilateral frontal; Figure 3b) resulted in an increased N100 amplitude. This change, however, was absent following 100\% iTBS ( $p>.025$; Figure 3c), and no other peaks showed any significant changes (all $p>$.025). We compared the TEPs between BL and 30-min post iTBS (T30), but no significant persistent effect remained (all $p>$.025). In order to evaluate the differences between conditions, iTBS-induced changes in TEP amplitude were calculated (post - pre) and compared. As our experimental design was not sham-controlled, this method of comparison would minimize the confounding factor (e.g., change over time unrelated to stimulation). We found that the change in $\mathrm{N} 100$ amplitude $(\Delta \mathrm{N} 100)$ was the largest with 75\% iTBS, but less following 100\% iTBS (75\% > 100\% iTBS: T5, $112-140 \mathrm{~ms}, p=.008$ ), which was observed in fronto-central sensors
(Figure 3d). Source estimation of N100 in these stimulation conditions supported the findings of the scalp-level analyses, where increased electrical activity was found in fronto-central region following $75 \%$ iTBS, but minimal change was seen following 100\% iTBS (Figure 3d). The differences were not apparent when these conditions were compared with 50\% iTBS (all $p>.025$ ), placing the strength of the aftereffect of $50 \%$ iTBS in the middle of $75 \%$ and $100 \%$ iTBS.

Given the implication of P60 and N100 peaks in excitatoryinhibitory balance, we explored the relationship between these peaks modulated by iTBS. Correlation analysis was conducted on the data combined across different conditions $(n=48)$ using the average of 3 fronto-central electrodes (FC1, FCz, FC2) as these electrodes were close to the stimulation, and often showed significant changes following iTBS. Spearman's rank correlation revealed a significant correlation between $\Delta$ P60 and $\Delta$ N100 at T5 $(r=-0.385, p=.007)$ and a trend toward significance at T30 $(r=-0.257, p=.077)$ (Figure 4).

iTBS-induced changes in TMS-evoked oscillations (averaged across all electrodes) are illustrated in Figure 5. We assessed whether different iTBS conditions altered TMS-evoked theta and gamma power (total activity: evoked + induced) in a similar fashion. The cluster-based permutation test revealed a significant increase in TMS-evoked theta power at T5 compared to BL in close proximity to the stimulation site following $75 \%$ iTBS $(p=.024)$, but not with $50 \%$ or $100 \%$ iTBS $(p>.025)$. Between conditions, the change in theta power ( $\Delta$ theta) was larger following $75 \%$ iTBS compared to $100 \%$ iTBS (75\% > 100\% iTBS: T5, $p=.020$; Figure $5 \mathrm{~d}$, top row). However, no prolonged theta change was observed at T30 (all $p>.025$ ).

Initially, TMS-evoked gamma power showed slightly different changes, with significantly decreased gamma power following $100 \%$ iTBS at T5 $(p=.023)$, which was most pronounced over the frontal sensors. On the other hand, 75\% iTBS exhibited nonsignificant increase in the frontal and parietal regions. Even though no significant differences between BL and T30 were observed in gamma frequency band in any stimulation conditions (all $p>.025$ ), between condition comparisons revealed the change in gamma power ( $\Delta$ gamma) was significantly different between $75 \%$ and 100\% iTBS at both T5 and T30, which resulted from polarity-specific changes following the two stimulation conditions. At T5, the difference was most pronounced over bilateral frontal sensors $(p=.006)$ and parieto-occipital sensors $(p=.015)$, and at T30, the differences were observed at left frontal $(p=.019)$ and left parietal region ( $p=.006$ ) (Figure $5 \mathrm{e}$, top row). Again, no significant differences were found between $50 \%$ and $75 \%$ iTBS, or $50 \%$ and $100 \%$ iTBS for changes in theta or gamma power (all $p>$.025).

Examination of evoked oscillations revealed that only 75\% iTBS significantly increased both theta ( $p=.019$, fronto-central) and gamma power ( $p=.016$, parieto-occipital) at T5, but not at T30. Neither 50\% nor 100\% iTBS showed any significant change in these frequency bands (all $p>$.025). For between condition comparisons, $\Delta$ theta showed significant difference between $75 \%$ and $100 \%$ iTBS at T5 (75\% > 100\% iTBS: $p=.009$, fronto-central) (Figure $5 \mathrm{~d}$, bottom row), but not in $\Delta$ gamma (Figure $5 \mathrm{e}$, bottom row). No significant differences in $\Delta$ theta or $\Delta$ gamma were found between $50 \%$ and $75 \%$ iTBS, or $50 \%$ and $100 \%$ iTBS at any time point (all $p>$.025). 
(a) $50 \%$ iTBS

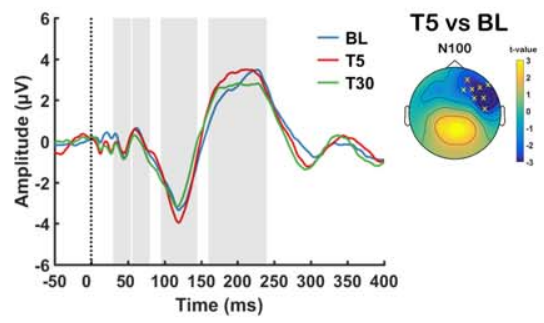

(b) $75 \%$ iTBS

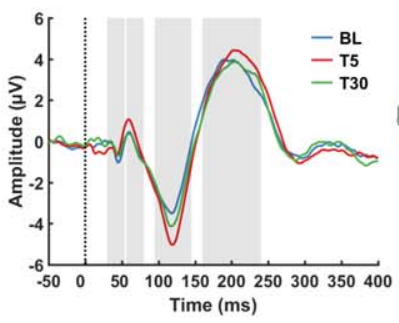

(c) $100 \%$ iTBS

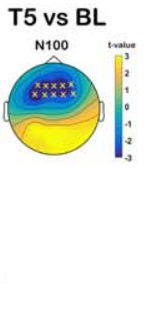

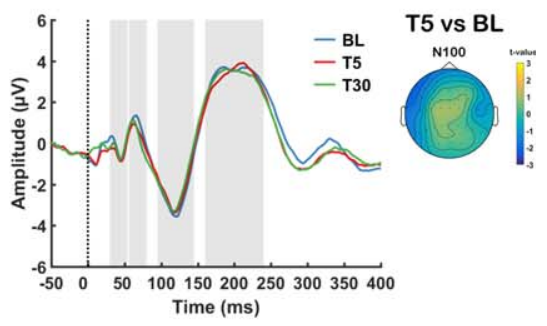

(d) $75 \%$ vs $100 \%$

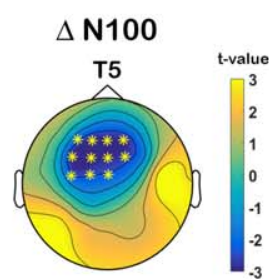

$75 \%$ iTBS

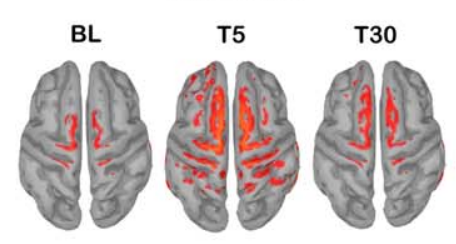

$100 \%$ iTBS

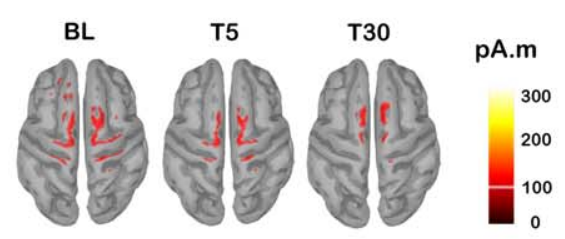

FIGURE 3 Assessment of transcranial magnetic stimulation (TMS)-evoked potentials (TEPs) before and after each stimulation condition [a: Intermittent theta-burst stimulation (iTBS) at 50\% rMT (50\% iTBS); b: iTBS at 75\% rMT (75\% iTBS); c: iTBS at $100 \%$ rMT (100\% iTBS)]. Grand average TEP waveforms before (BL: blue), 5-min post (T5: red) and 30-min post (T30: green) iTBS at FCz electrode for each stimulation conditions, with significant differences across the scalp illustrated in topoplots. (d) Global scalp differences of iTBS-induced change in N100 amplitude (TEP $\Delta$ N100) between 75\% and 100\% iTBS at T5 and Minimum Norm Estimates (MNEs) of the source level activity at the cortex for the N100 peak. Asterisks and ' $\mathrm{X}$ ' $\mathrm{s}$ on topoplots indicate significant clusters between comparisons (cluster-based statistics, ${ }^{*} p<.01,{ }^{x} p<.025$ ) [Color figure can be viewed at wileyonlinelibrary.com]

Exploratory analyses including all dimensions of the data (time $x$ frequency $\times$ space) were conducted to investigate iTBS-induced changes in all oscillatory bands and time windows. However, we found no significant differences within or between stimulation conditions (all $p>$.025). Subgroup analysis on alpha $(8-12 \mathrm{~Hz})$ and beta $(13-29 \mathrm{~Hz})$ frequency bands [time $\times$ alpha/beta (frequency range averaged prior to cluster-statistics) $\times$ space] also resulted in no significant changes (all $p>$.025).

A recent study using prefrontal-parietal paired associative stimulation (PAS) protocol demonstrated increased cortical responses to TMSinduced plastic effects in subjects with higher gamma power (Casula, Pellicciari, Picazio, Caltagirone, \& Koch, 2016a), and we explored whether observed changes in theta and gamma power had any relationship with each peak of interest. Spearman's rank correlation revealed significant correlations between $\Delta$ gamma and $\Delta \mathrm{P} 60$ (T5: $r=0.353, p=.014)$ and $\Delta \mathrm{N} 100$ (T5: $r=-0.347, p=.016$; T30: $r=-0.326, p=.024)$, and between $\Delta$ theta and $\Delta$ P200 (T5: $r=0.597$, $p=.001$ ) (Supporting Information Figure S1), but not with $\Delta$ N45 (all $p>$.05). These findings indicate increased amplitude of multiple peaks are associated with stronger oscillatory activity in either theta or gamma range. This is in agreement with previous findings for PAS, but also demonstrates the specificity to theta and gamma for iTBS.

\subsection{The effect of different iTBS intensities on working memory neurophysiology}

Before we examined the effect of iTBS on ERPs during WM task, the effect of memory load on the ERPs was first established in our dataset

\section{T5}

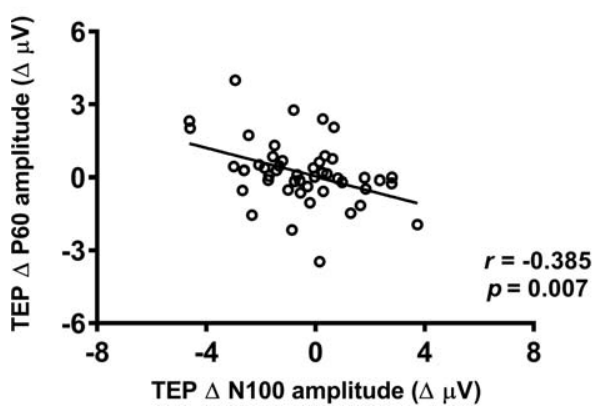

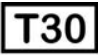

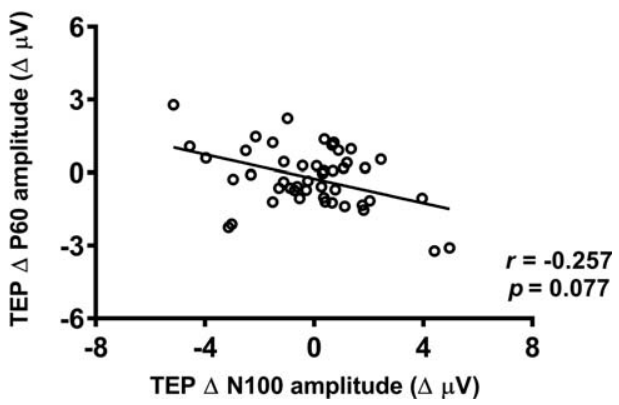

FIGURE 4 Correlation between intermittent theta burst stimulation (iTBS)-induced changes in transcranial magnetic stimulation (TMS)evoked potential (TEP) N100 and P60 amplitude 
(a) $50 \%$ iTBS

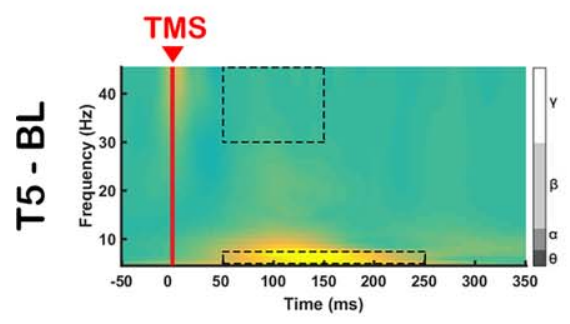

(b) $75 \%$ iTBS

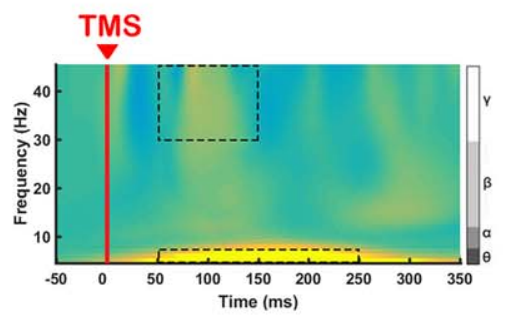

(c) $100 \%$ iTBS

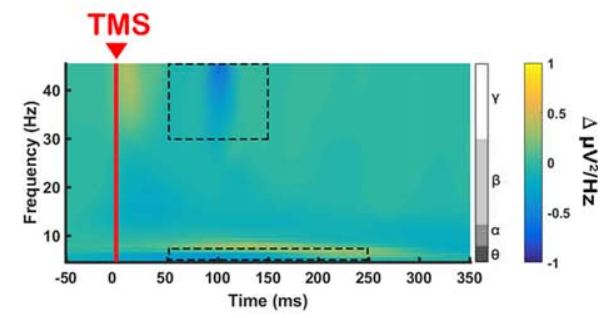

\section{$75 \%$ vs $100 \%$}

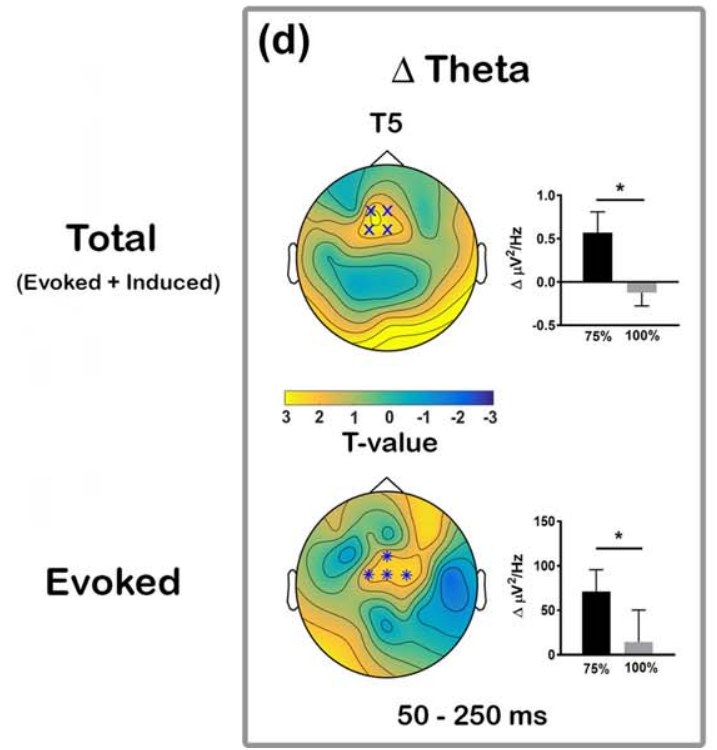

(e)

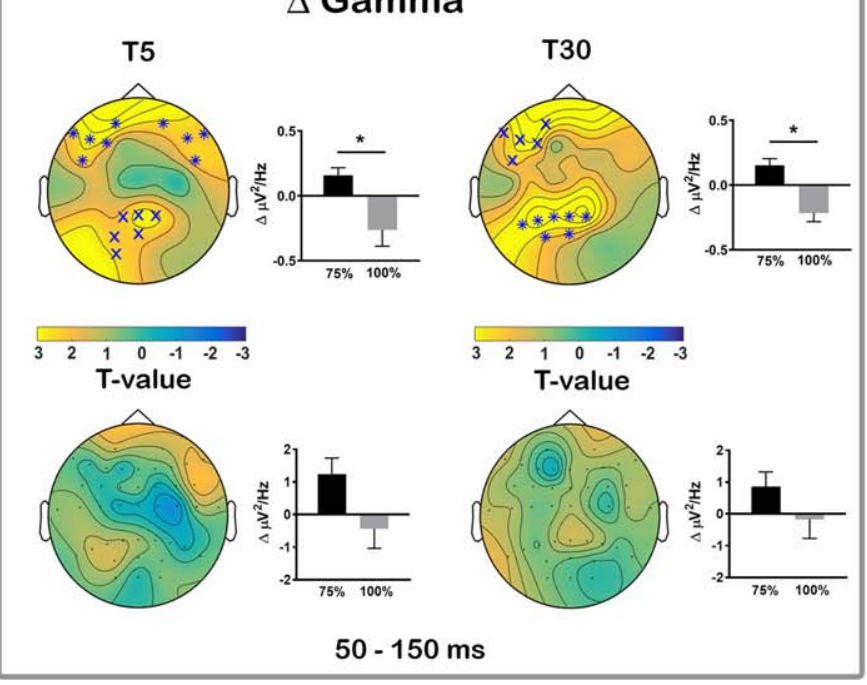

FIGURE 5 Comparison of transcranial magnetic stimulation (TMS)-evoked oscillations in iTBS-induced changes [a: Intermittent theta-burst stimulation (iTBS) at 50\% rMT (50\% iTBS); b: iTBS at 75\% rMT (75\% iTBS); c: iTBS at 100\% rMT (100\% iTBS)]. Grand average timefrequency plots are illustrated using average of all electrodes and displayed the difference between baseline and T5 ( $\Delta$ power; T5 - BL). Dotted boxes represent time-frequency windows for gamma (50-150 ms) and theta (50-250 ms) bands where statistical analyses were conducted. Comparison between $75 \%$ and $100 \%$ iTBS conditions in (d) $\Delta$ theta at T5 and (E) $\Delta$ gamma at T5 and T30 across the scalp. Both total power (evoked + induced; top row) and evoked power alone (bottom row) were examined separately. Asterisks and ' $X$ ' $s$ on topoplots indicate significant clusters between comparisons (cluster-based statistics, ${ }^{*} p<.01,{ }^{x} p<.025$ ). Bar graphs were plotted using the values extracted from the significant sensors (when not significant, using same sensors as total power) to examine the directional changes [Color figure can be viewed at wileyonlinelibrary.com]

using BL measurement (Supporting Information Figure S2A). To test if iTBS-induced changes measured by TEPs were consistent with electrophysiology recordings during WM task, we investigated the ERPs during 2-back and 3-back tasks in a similar manner to TEPs. Supporting the outcome in the TEPs measurement, 75\% iTBS significantly increased the amplitude ERP N200 (198-218 ms, $p=.022$, fronto-central) during 2-back task (Figure 6a). The change in N200 amplitude (ERP $\Delta$ N200) was the largest with $75 \%$ iTBS compared to $100 \%$ iTBS (ERP $\Delta$ N200: 190-228 ms, $p=.018$, fronto-central). Source estimation of ERP N200 in these stimulation conditions revealed activity of parieto-occipital origin, and 75\% iTBS resulted in increased activity including fronto-central region, whereas minimal change was observed following 100\% iTBS (Figure 6c). Similar to TEPs, the differences were not significant when these conditions were compared with 50\% iTBS (all $p>$.025). During the 3-back task, cluster-based statistics revealed $50 \%$ and $75 \%$ iTBS, but not $100 \%$ iTBS, resulted in significant differences between BL and T15 in ERP P300 amplitude, which was observed over anterior (50\% iTBS: $310-333 \mathrm{~ms}, p=.023$; 75\% iTBS: 315-343 ms, $p=.015$ ) sensors, indicating increased amplitude following 50\% and 75\% iTBS (Figure 6b). However, no significant differences were seen between different stimulation conditions in P300 or any other peaks (all $p>$.025).

As both the TEP N100 and cognitive task related N200 peaks have been associated with inhibitory mechanisms [TEP N100 (Farzan et al., 2013; Premoli et al., 2014; Rogasch et al., 2015); ERP N200 (Aron, 2007; Kopp, Rist, \& Mattler, 1996; Sasaki, Gemba, \& Tsujimoto, 1989)], correlation analysis was performed on the data combined across different conditions $(n=45)$ using the average of 3 frontocentral electrodes (FC1, FCz, FC2). These electrodes were close to the stimulation, and the significant changes were most often observed in these electrodes across different measures. Spearman's rank correlation revealed TEP $\Delta$ N100 amplitude following iTBS (T5) correlated 
(a) 2-back
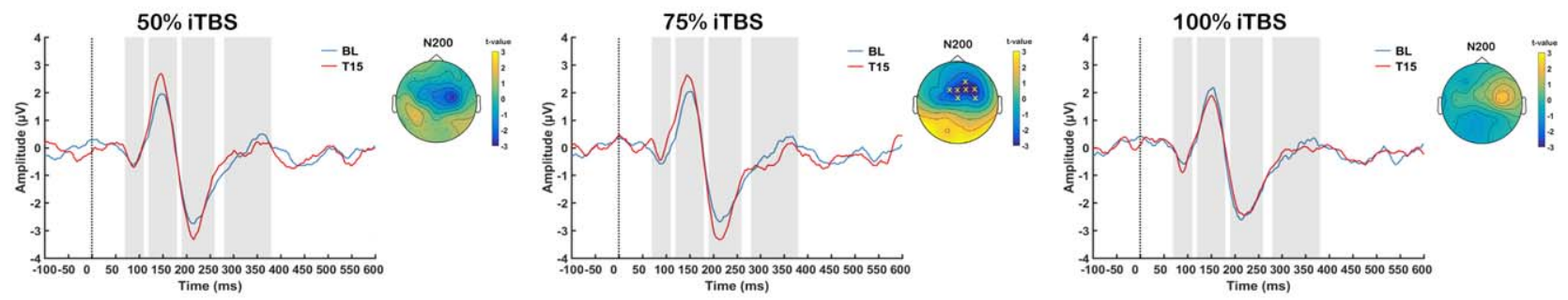

(b) 3-back
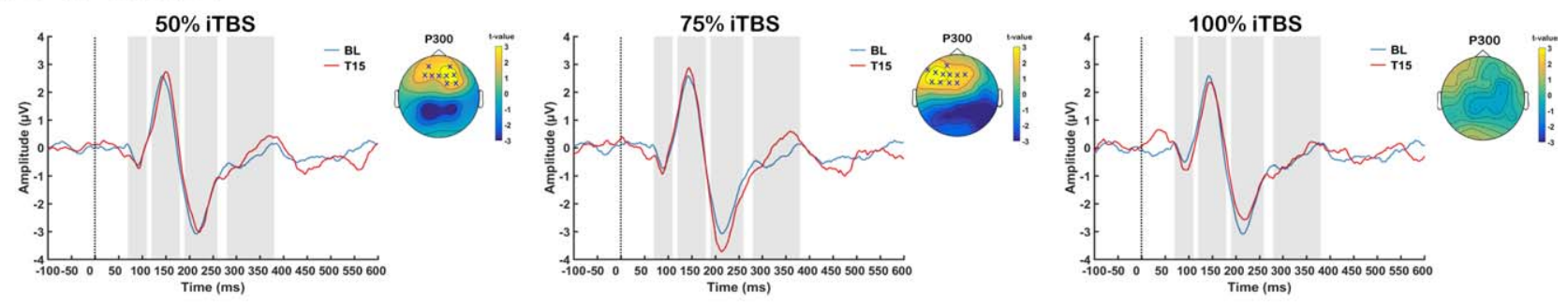

(c) $75 \%$ vs $100 \%$
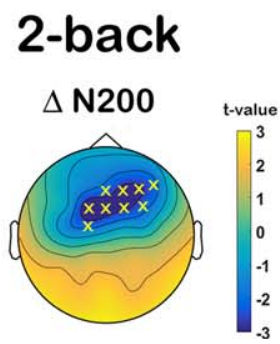

$75 \%$ iTBS

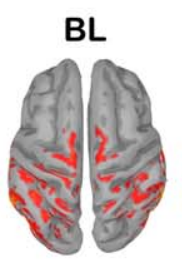

T15

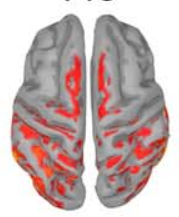

$100 \%$ iTBS

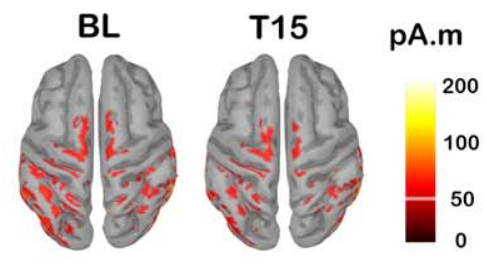

FIGURE 6 Effect of different intensities of intermittent theta-burst stimulation (iTBS) on the event related potentials (ERPs) during working memory tasks. Grand average ERP waveforms at baseline (BL: blue) and 15-min post (T15: red) iTBS at FCz electrode for each stimulation conditions (50\%, 75\%, and 100\% iTBS) in (a) 2-back and (b) 3-back tasks, with significant differences across scalp shown in topoplots. (c) Global scalp differences of iTBS-induced change in N200 amplitude (ERP $\Delta$ N200) during 2-back task between $75 \%$ and $100 \%$ iTBS at T15 and Minimum Norm Estimates (MNEs) of the source level activity at the cortex for the N200 peak. ' $\mathrm{X}$ ' $\mathrm{s}$ on topoplots indicate significant clusters between comparisons (cluster-based statistics, ${ }^{x} p<.025$ ) [Color figure can be viewed at wileyonlinelibrary.com]

with ERP $\Delta$ N200 amplitude during 2-back task (T15) ( $r=0.572$, $p=.001$; Figure 7). We also explored if TEP $\Delta$ N100 correlated with ERP $\Delta$ P150 during 2-back task, or ERP $\Delta 300$ during 3-back task, however, no significant correlations were found (all $p>.05$ ). The correlation between TEP $\Delta$ N100 and ERP $\Delta$ N200 during 2-back task supports the evidence that iTBS alters cortical inhibition in human prefrontal cortex at subthreshold intensities.

We also assessed the effect of different iTBS intensities on theta and gamma oscillations during WM. The effect of memory load on these oscillations was again examined using BL measurement (Supporting Information Figure S2B). As N-back task involves continuous mix of encoding, updating and maintaining of the letters, we divided each trial into two blocks-during letter presentation (50-450 ms: encoding) and after letter presentation (550-950 ms: maintenance).

To assess whether iTBS was able to modulate these frequency bands during WM task, both theta and gamma power were compared across time, and the changes between conditions. During letter presentation, iTBS did not change any frequency band during 2-back task (all $p>$.025). However, during 3-back task, significant increases in theta power were found at T15 compared to BL following both 50\% ( $p=.013$, right prefrontal) and $75 \%$ iTBS ( $p=.023$, left prefrontal), but not $100 \%$ iTBS, indicating theta oscillations increased with subthreshold intensities. When $\Delta$ theta power were compared between conditions, differences were observed only between $75 \%$ and $100 \%$ iTBS (75\% > 100\% iTBS, $p=.022$ ) over left prefrontal sensors (Figure 8a, top row). While gamma power changes were not observed in any stimulation conditions in any $\mathrm{N}$-back task, $\Delta$ gamma was significantly different between $75 \%$ and $100 \%$ iTBS (75\% > 100\% iTBS, $p=.022)$ over left posterior sensors during 2-back task (Figure 8b, top row), but not during 3-back task. These findings suggest that iTBS differentially modulates cortical oscillations during letter presentation across task loads. After the letter presentation, however, iTBS resulted in no change in either theta or gamma band during either memory task (all $p>$.025), which suggests iTBS was not able to alter the processing involved in maintenance of memory.

Analysis of evoked oscillations resulted in a different pattern to the evoked oscillatory activity during TMS-EEG. We found no 


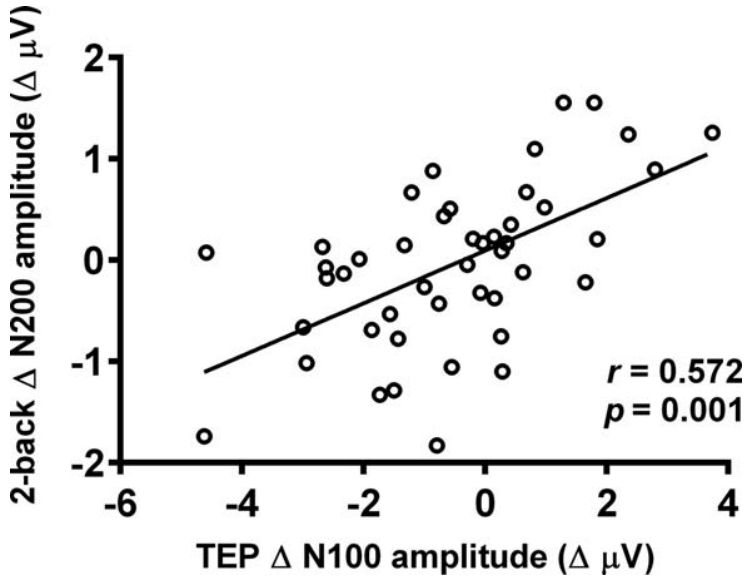

FIGURE 7 Correlation between iTBS-induced changes in TMSevoked potential (TEP) N100 amplitude and 2-back task related N200 amplitude

significant differences in any frequency bands within or between conditions in any task (all $p>$.025). However, we observed nonsignificant increase in theta power following 75\% iTBS, which was absent following $100 \%$ iTBS in 3-back task (Figure 8a, bottom row). We were unable to detect any changes in gamma power in the evoked activity in 2-back task (Figure 8b, bottom row).

Similar to TMS-EEG time-frequency analyses, exploratory analyses including all dimensions of the data (time $\times$ frequency $\times$ space) were conducted to investigate iTBS-induced changes in all oscillatory bands and time windows. However, no significant differences were found within or between stimulation conditions both in 2-back and 3-back task (all $p>$.025). In addition, we found no significant differences in alpha or beta frequency band (all $p>.025$ ).

We tested if TMS-evoked oscillations ( $\Delta$ theta and $\Delta$ gamma) shared similar mechanisms to $\mathrm{N}$-back task related oscillations ( $\Delta$ theta with 3-back, $\Delta$ gamma with 2-back). For gamma oscillations, average of 3 left/mid parietal electrodes (P3, P1, Pz) were used for correlation analysis as these were found significant in cluster-based analysis of both TMS-evoked and 2-back task. Spearman's rank correlation revealed the TMS-evoked $\Delta$ gamma power following iTBS (T5) correlated with $\Delta$ gamma power during 2-back task (T15) $(r=0.420$, $p=.004$; Figure 9). For theta oscillations, average of 3 fronto-central electrodes (FC1, FCz, FC2) were used. However, no significant correlation was found in $\Delta$ theta power between TMS-evoked and 3-back task $(r=0.078, p=.609)$.

\subsection{The effect of iTBS intensity on working memory}

$\mathrm{N}$-back WM performance (accuracy $d^{\prime}$, reaction time and effect sizes (Hedges' g [Hedges \& Olkin, 1985]) is shown in Table 1.

\subsection{1 $\mid$ Performance at baseline}

Initial statistical analysis was conducted on pre-iTBS (BL) data (combined across sessions, $n=45$ ) to determine if WM performance differed between different memory load conditions (2-back vs 3-back) in accuracy $\left(d^{\prime}\right)$ and reaction time. The Wilcoxon signed-rank tests revealed $d^{\prime}$ scores decreased $[Z=-5.108, r=-0.35, p=.001$ (3-back$<2$-back)] and reaction times increased $[Z=2.523, r=0.18, p=.012$

\section{$75 \%$ vs $100 \%$}

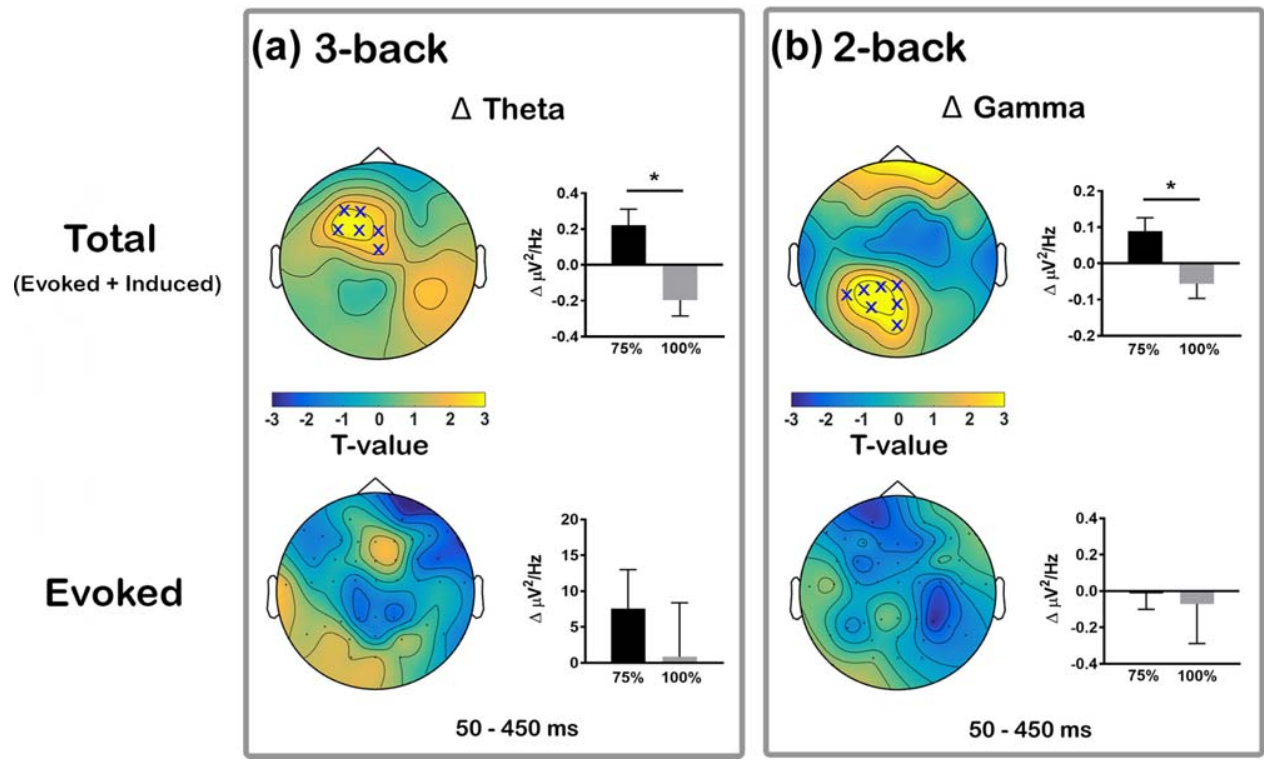

FIGURE 8 Comparison of intermittent theta-burst stimulation (iTBS)-induced changes in theta and gamma oscillations during different working memory tasks between $75 \%$ and $100 \%$ iTBS conditions. Significant differences in iTBS-induced change in (a) $\Delta$ theta power during 3-back task and in (b) $\Delta$ gamma power during 2-back task across the scalp. Both total power (evoked + induced; top row) and evoked power alone (bottom row) were examined separately. ' $X$ ' $s$ on topoplots indicate significant clusters between comparisons (cluster-based statistics, $x_{p<.025)}$. Bar graphs were plotted using the values extracted from the significant sensors (when not significant, using same sensors as total power) to examine the directional changes [Color figure can be viewed at wileyonlinelibrary.com] 


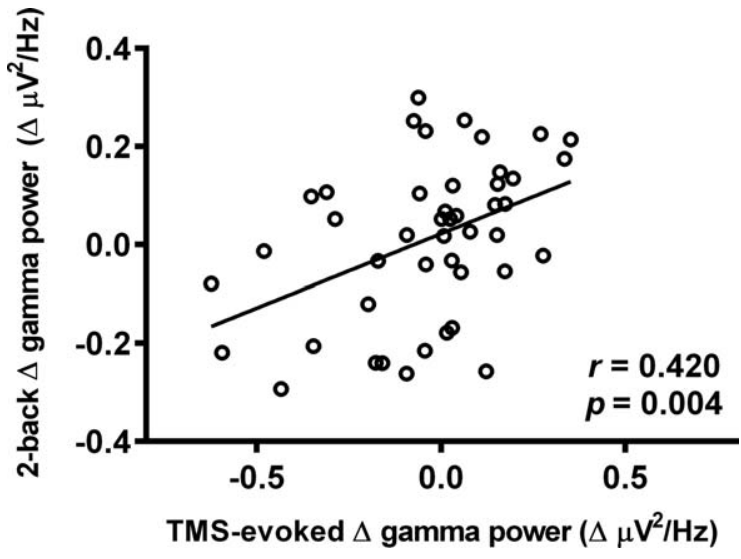

FIGURE 9 Correlation between iTBS-induced changes in TMSevoked gamma power and 2-back task related gamma power

(3-back $>2$-back)] with increasing WM load. We also conducted order effect analysis to confirm the effectiveness of the counter-balancing of stimulation conditions. Friedman's ANOVA showed no significant session effects in either $d^{\prime}$ (2-back: $x^{2}=0.037, p=.982$; 3-back: $\left.x^{2}=0.036, p=.982\right)$ or accurate reaction time (2-back: $x^{2}=3.448$, $p=.178$; 3-back: $x^{2}=1.793, p=.408$ ) for $W M$ tasks at baseline measure.

\subsection{2 | Performance following iTBS}

Following $75 \%$ iTBS there was a significant decrease in reaction time (Wilcoxon signed rank test; $p=.031$ ) of small-to-moderate effect size (-0.42) during 3-back task. No other stimulation conditions showed any significant differences in WM performance (Wilcoxon signed rank test; all $p>.05)$.

\subsection{3 | Comparison pre- and post-iTBS}

When compared across conditions using the change-from-baseline scores (post - pre; $\Delta$ ), we could not detect any significant differences in reaction time or $d^{\prime}$ (Friedman's ANOVA; all $p>.05$ ).

We next tested if physiological changes were related to improved reaction time following $75 \%$ iTBS. The correlation analyses were performed between significant changes observed following 75\% iTBS in TMS-EEG ( $\Delta$ N100, $\Delta$ theta, $\Delta$ gamma) and during 3-back task ( $\Delta$ P300, $\Delta$ theta) against $\Delta$ reaction time in $75 \%$ iTBS condition during 3-back task. However, there was no significant correlation between any change in physiological measure and 3-back reaction time (all $p>.05$ ).

\section{5 | Control analyses}

\subsection{1 | Assessment of carryover effect}

Studies have used 72 hours as a wash-out period for various noninvasive brain stimulation techniques (Chung et al., 2017; Hameed et al., 2017; Hill et al., 2017; Kumpulainen, Mrachacz-Kersting, Peltonen, Voigt, \& Avela, 2012; Vossen, Gross, \& Thut, 2015). To test if 72 hours were sufficient to avoid carryover effect of iTBS, baseline (BL) neurophysiological data were rearranged in the order of session and statistical analyses were performed on both TMS-EEG and N-back EEG data.

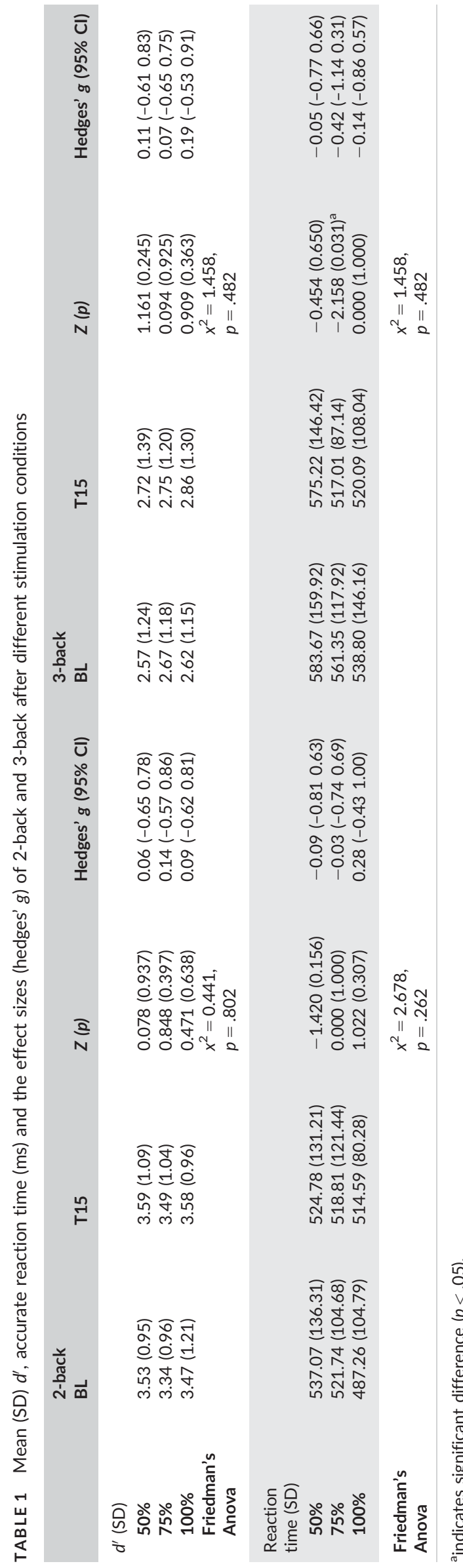


We found no significant differences between any sessions in any neurophysiological data (all $p>$.025) (Supporting Information Figure S3), suggesting the absence of carryover effect.

\subsubsection{Secondary analyses of sham condition}

Neurophysiology

No significant differences were observed in the amplitudes of any TEPs or ERPs (both the 2-back and 3-back tasks) across time (Supporting Information Figure S4A-C), as well as in the oscillatory power in these measures (all $p>$.025) following sham iTBS.

When compared to active conditions, cluster-based permutation test (independent t-test) between 75\% iTBS and sham iTBS showed a significant difference in TEP $\Delta$ N100 (75\% > sham iTBS: T5, 109-140 $\mathrm{ms}, p=.009$; fronto-central sensors). Similarly, a significantly larger TMS-evoked theta power was seen following $75 \%$ iTBS compared to sham (T5, $p=.019$, fronto-central sensors). During the 2-back task, a significantly larger gamma power was observed following 75\% iTBS compared to sham ( $p=.024$; parietal sensors) during the letter presentation. During the 3-back task, a significantly larger alpha power was seen following $75 \%$ iTBS compared to sham ( $p=.024$; left prefrontal sensors) during the maintenance period (550-950 ms) (Supporting Information Figure S4D). A comparison between 100\% iTBS and sham yielded a significantly difference only in TMS-evoked gamma power (100\% < sham iTBS: T30, $p=.020$; left-prefrontal sensors) (Supporting Information Figure S4E). No other change in peaks or oscillatory power showed any significant differences either in TMS-EEG or N-back EEG (all $p>$.025).

\section{Behaviour}

Table 2 summarises the $\mathrm{N}$-back performance and the effect size of sham control iTBS and its comparison to active stimulation conditions. While no significant differences were found between BL and T15 following sham stimulation in either $d^{\prime}$ or accurate reaction time for both the 2-back and the 3-back tasks (Wilcoxon signed rank test; all $p>.05$ ), between condition comparisons using the Mann-Whiney $U$ test indicated that 75\% iTBS elicited a greater improvement in accurate reaction time than sham stimulation during the 3-back task $(U=44$, $p=.025)$.

\subsubsection{Association of alpha power and reaction time}

Increased alpha power has been associated with faster reaction time in a motor task (Moore, Gale, Morris, \& Forrester, 2008) and in working memory (Bonnefond \& Jensen, 2012; Nenert, Viswanathan, Dubuc, \& Visscher, 2012). Similarly, $\Delta$ alpha (Figure 10a,b) resembled closely to $\Delta$ accurate reaction time (Figure 10c). Therefore, we explored if these changes were related. Correlation analysis was conducted on the data combined across active iTBS conditions $(n=45)$ using the average of all electrodes. Spearman's rank correlation revealed the $\Delta$ alpha power significantly correlated with $\Delta$ accurate reaction time during 3-back task ( $r=-0.603, p=.001$ ) (Figure 10d), suggesting increased alpha power leads to faster reaction time.

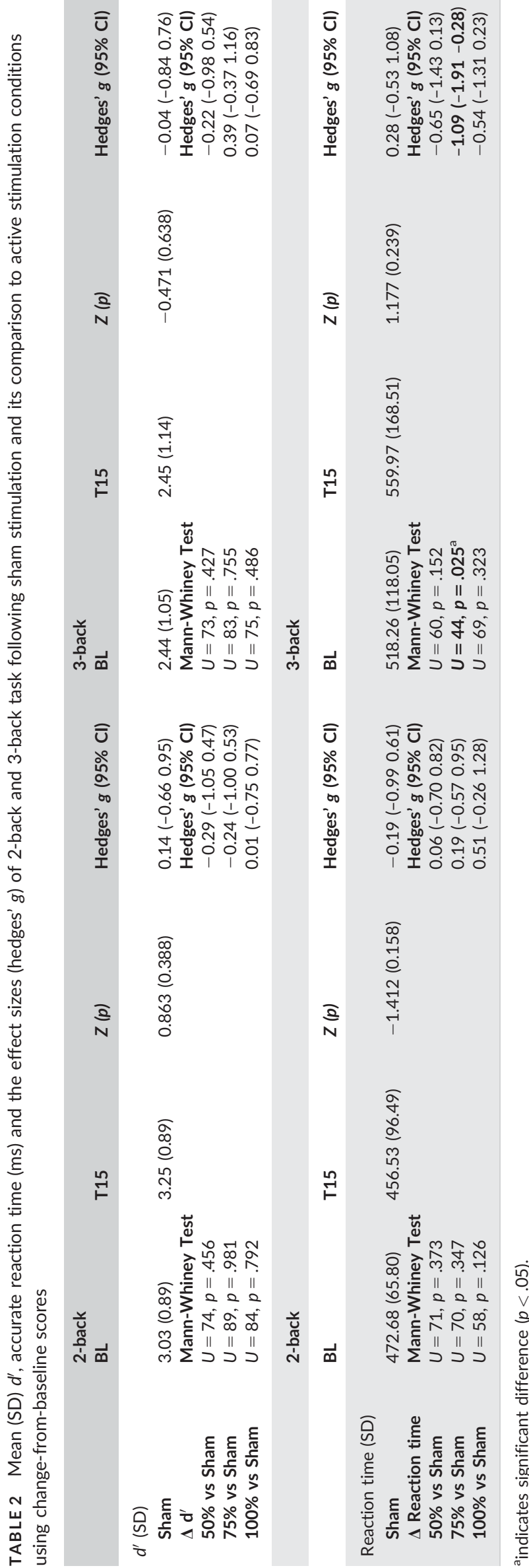




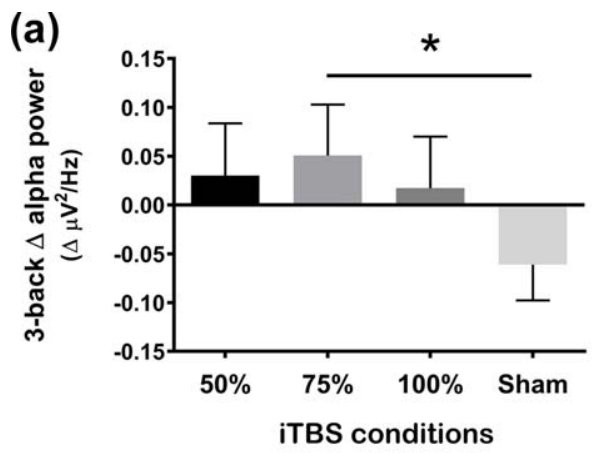

(b) $75 \%$ iTBS vs Sham

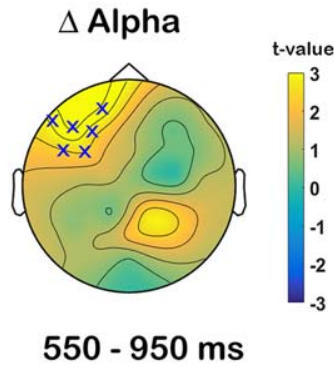

(c)

(c)
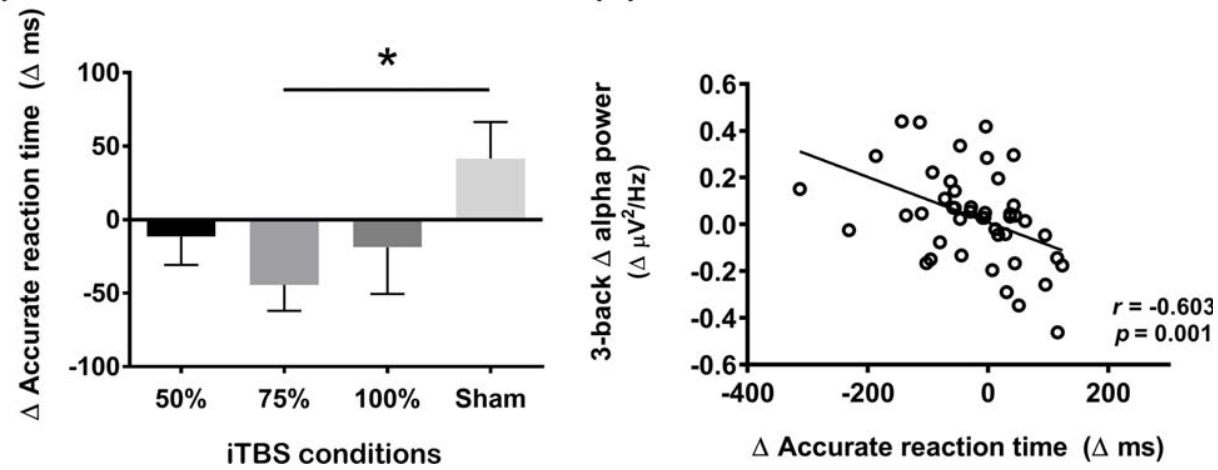

FIGURE 10 Comparison of the change $(\Delta)$ in alpha power between different stimulation condition during the 3-back task and its association to working memory performance. (a) Comparison of global scalp $\Delta$ alpha power between $50 \%, 75 \%, 100 \%$ and sham intermittent theta burst stimulation (iTBS) and (b) scalp map representing $t$-values for the significant differences (cluster-based statistics, ${ }^{x} p<.025$ ). (c) Comparison of $\Delta$ accurate reaction time between different stimulation condition and (D) correlation between $\Delta$ alpha power and $\Delta$ accurate reaction time following active iTBS. Error bars indicate standard error of means (SEM) [Color figure can be viewed at wileyonlinelibrary.com]

\section{4 | DISCUSSION}

This study examined the link between iTBS intensity and LTP-like neural plasticity, and the association to neurophysiological and behavioural metrics of WM in the prefrontal cortex. The data indicate an inverse $\mathrm{U}$ shaped relationship between iTBS intensity and neurophysiological changes following single-pulse TMS and during working memory, whereby these effects were maximal at an intermediate intensity of 75\% rMT. However, no differences in working memory performances were seen between active conditions. The plastic effects correlated with changes in neurophysiological aspects of cognition (ERPs), however these changes did not have a direct relationship with the behavioural outcomes of the WM task (accuracy and reaction time). Instead, iTBS-induced change in alpha power during the 3-back task demonstrated close association to the change in reaction time. The data suggest using subthreshold intensities is important in order to achieve desirable after-effects following iTBS in the prefrontal cortex, and highlight potential benefits in the application of iTBS for clinical treatment.

\subsection{Influence of iTBS intensity on plastic effects in DLPFC}

iTBS modulated N100 amplitude, and the increase in this component was maximal when iTBS was delivered at 75\% rMT compared to lower (50\% rMT) or higher intensity (100\% rMT). These findings raise interesting aspects of the relationship between intensity and plasticity induction. Increased N100 following iTBS over prefrontal cortex is in line with previous studies which also showed modulation of this component following iTBS (Chung et al., 2017; Harrington \& HammondTooke, 2015) or cTBS (Harrington \& Hammond-Tooke, 2015; Huang \& Mouraux, 2015), however, opposite outcome (i.e., decreased following iTBS, increase following cTBS) has also been described in cerebellar stimulation (Casula et al., 2016b). The N100 deflection is considered to be the most prominent and robust TMS-EEG component and is understood to have the greatest inter-individual and inter-session reproducibility compared to other TEPs both in motor and prefrontal cortex (Lioumis, Kicic, Savolainen, Makela, \& Kahkonen, 2009). The N100 is also considered to have a high degree of sensitivity to small changes in cortical excitability (Nikulin, Kicic, Kahkonen, \& Ilmoniemi, 2003). These factors enhance the value of $\mathrm{N} 100$ as a marker of cortical processing in basic and clinical research, and make it ideal for exploration of the effects of TMS plasticity paradigms (Chung et al., 2015b; Ilmoniemi \& Kicic, 2010; Noda et al., 2016). Recent studies have provided evidence that N100 may also be associated with $\mathrm{GABA}_{\mathrm{B}}$-mediated postsynaptic inhibition in motor (Farzan et al., 2013; Premoli et al., 2014; Rogasch et al., 2013a) and prefrontal (Rogasch et al., 2015) cortex. These findings raise the prospect of current data reflecting an increase in cortical inhibition following iTBS. This account is difficult to reconcile with the absence of effects of iTBS on $\mathrm{GABA}_{B}$-mediated inhibitory measures such as long intracortical inhibition (LICI) (Goldsworthy, Pitcher, \& 
Ridding, 2013; Suppa et al., 2008) or the cortical silent period (Brownjohn, Reynolds, Matheson, Fox, \& Shemmell, 2014; Di Lazzaro et al., 2011) in the motor cortex. However, we did recently observe an increase in $\mathrm{LICl}$ of theta oscillations which correlated with increased N100 amplitude following iTBS over DLPFC (Chung et al., 2017), supporting possible modulation of cortical inhibition following stimulation.

The present data did not show a significant increase in P60 amplitude following iTBS. Recent studies suggest that $\mathrm{P} 60$ provides a marker of neural excitability (Cash et al., 2017b; Hill et al., 2017). It should be noted that the SNR for P60 is substantially lower than for N100, and the current protocol with $\sim 47$ single TMS pulses may have been insufficient to capture significant changes. The data, however, demonstrated evidence of a relationship between the change in amplitude of N100 and P60 following iTBS. If N100 is related to inhibition, and P60 to neural excitability, this finding suggests that the change in excitation was balanced by a similar change in inhibition, maintaining the excitatory-inhibitory balance following iTBS. This is in agreement with the concept of homeostatic plasticity mechanisms involving a dynamic adjustment of excitatory and inhibitory circuits (Turrigiano \& Nelson, 2004). In summary, it appears that the most reliable TMS-EEG metric of plasticity is the modulation of N100 amplitude and iTBS-induced change in this component was greatest at an intermediate intensity of 75\% rMT.

The relationship between iTBS intensity and the level of plasticity induction is likely explained by the unique mechanistic features underlying iTBS. Typically, the propensity for LTP-like effects increases with increasing intensity (Artola, Brocher, \& Singer, 1990; Cash et al., 2017a), whereby greater postsynaptic depolarisation leads to higher levels of N-methyl-D-aspartate receptor (NDMA-R) activation, and consequently regulating the processes leading to LTP (Luscher \& Malenka, 2012). A similar relationship has been demonstrated across a range of noninvasive NMDA-R dependent brain stimulation protocols in human (Batsikadze et al., 2013; Cash et al., 2017a; Doeltgen \& Ridding, 2011; Moliadze, Atalay, Antal, \& Paulus, 2012). However, the present findings demonstrate an exception to this relationship, showing an inverse $U$-shaped influence of stimulus intensity on plastic effects. This may be explained by the unique temporal aspects that underlie the fundamental mechanism of TBS (Larson \& Munkacsy, 2015). It is thought that the robust after-effect of TBS is achieved through targeting a late period of presynaptic $G_{A B A}$-mediated disinhibition, which may itself help sustain the theta rhythm (Davies, Starkey, Pozza, \& Collingridge, 1991; Larson \& Munkacsy, 2015; Mott \& Lewis, 1991). More specifically, stimulation elicits both postsynaptic $G_{A B A}$-mediated inhibition (inhibitory postsynaptic potentials) and presynaptic $G_{A B}$ autoreceptor-mediated disinhibition (temporary blockade of further GABA release). It has been shown that presynaptic disinhibition outlasts postsynaptic inhibition, resulting in a late temporal window ( 200 ms) during which disinhibition dominates (Deisz, 1999; Otis, De Koninck, \& Mody, 1993) and plasticity induction is enhanced (Davies \& Collingridge, 1996; Larson \& Lynch, 1986; Mott \& Lewis, 1991; Pacelli, Su, \& Kelso, 1989). Delivery of stimulus bursts at this interval (i.e., TBS) results in a rapid induction of plastic effects (Davies et al., 1991; Mott \& Lewis, 1991). A similar late phase of disinhibition has recently been described in humans at $\sim 200$ ms latency (Cash, Ziemann, Murray, \& Thickbroom, 2010), during which excitability (Cash, Ziemann, \& Thickbroom, 2011) and plasticity induction were enhanced (Cash, Murakami, Chen, Thickbroom, \& Ziemann, 2016). Importantly, the latency of this period increases with increasing stimulus intensity (Cash et al., 2010) and stimulation outside this window does not result in plastic effects in humans (Cash et al., 2016) or animals (Larson \& Munkacsy, 2015). Consequently, higher TBS intensities may miss this plastic window. This unique plasticity mechanism may account for the inverse U-shaped relationship between intensity and plasticity observed in this study.

\subsection{The effect of iTBS on neural oscillations is modulated by stimulus intensity}

The spectral characteristics elicited by single-pulse TMS are commonly modulated following TBS in a manner that may depend on the area being stimulated. Cerebellar stimulation (iTBS and cTBS) were found to modulate alpha and beta power (Casula et al., 2016b), while cTBS of motor cortex produced modulation of theta, alpha and beta power (Vernet et al., 2013). In the prefrontal cortex, polarity-specific changes in TMS-evoked theta power (increase following iTBS, decrease following cTBS) were demonstrated (Chung et al., 2017), and modulation of theta and gamma power were also observed in a resting EEG study (Wozniak-Kwasniewska, Szekely, Aussedat, Bougerol, \& David, 2014), suggesting TBS may be targeting the natural frequency of oscillations in the stimulated region. Our data indicate the additional dimension of iTBS intensity in modulating these spectral changes. Theta power was increased following iTBS, consistent with our previous study (Chung et al., 2017), and this effect was maximal at 75\% rMT. Previous findings in relation to gamma power have been somewhat inconsistent, showing no change (Chung et al., 2017), or an increase following cTBS (Vernet et al., 2013), and this may relate to low SNR of gamma and/or discrepancies in analysis methods such as the total power vs evoked power, and the level of spatial dynamics (region of interest vs global scalp analysis). Here, we examined both total and evoked activity, and the analysis of total power provided additional information about the spread of activity following iTBS in distant yet interconnected regions. In the present study, the direction of change in TMS-evoked gamma was further shown to depend on the intensity of the stimulation (increase with $75 \%$ iTBS, decrease with 100\%), and this change remained significant at T30. This was an interesting observation as iTBS on rat cortex also resulted in long-lasting gamma power increase (Benali et al., 2011), and this finding may indicate that the persistent difference in after-effects of iTBS could be observed in the gamma frequency band in humans. The increase in theta and gamma power following iTBS at 75\% rMT would seemingly suggest that this intensity might be advantageous for enhancing performance on cognitive tasks.

\subsection{Relationship to neurophysiological and behavioural metrics during cognitive performance}

Similar to TMS-EEG findings, iTBS modulated neurophysiological metrics during the performance of the cognitive task in an intensity- 
dependent manner. With 75\% rMT and 50\% rMT to some extent, iTBS increased N200 amplitude in the 2-back task, and P300 amplitude in the 3-back task, while no changes were evident at a higher intensity. N200 has been linked to executive control (Kopp et al., 1996) and cognitive and inhibitory processing (Folstein \& Van Petten, 2008; Sasaki et al., 1989; Schmajuk, Liotti, Busse, \& Woldorff, 2006). The change in ERP N200 amplitude correlated with plastic changes in TEP N100 amplitude, suggesting that these may be modulated by iTBS in a similar manner or have a degree of functional overlap. This link was not present with ERP P150 or ERP P300, further strengthening the selective link for possible inhibitory processing involved in two different measures following iTBS. In the frequency domain, frontal theta power was enhanced following 75\% iTBS during the 3-back task. There was also a trend for an increase in parietal gamma power during 2-back WM task, which was maximal following iTBS at 75\% rMT. These results are consistent with a maximal effect of iTBS at 75\% rMT observed in TMS EEG data. A significant correlation between the change in TMS-evoked gamma power and event-related gamma power during 2-back task provides further evidence of a relationship between the neural elements modulated by TBS, probed by single-pulse TMS and functionally recruited during a WM task. These results support the notion that iTBS can likely enhance the neurophysiological mechanisms mediating working memory (Hoy et al., 2016), and does so in an intensity-dependent manner. Theta and gamma oscillations are important in WM (Howard et al., 2003; Hsieh \& Ranganath, 2014) and these oscillation frequencies are targeted by iTBS. The involvement of fronto-parietal network control system (Dosenbach, Fair, Cohen, Schlaggar, \& Petersen, 2008) is also supported by the observation of the influence of TBS on visuospatial attention (Xu et al., 2013) and in WM task (Hoy et al., 2016).

We observed a significant correlation between $\Delta$ alpha power and $\Delta$ reaction time following active iTBS conditions during the maintenance period of the 3-back task. Alpha power has been associated with the gating and maintenance of relevant information during working memory (Manza, Hau, \& Leung, 2014), and protects against distractions (Bonnefond \& Jensen, 2012). Alpha power decreases with increasing load in the N-back task (Chen \& Huang, 2015; Scharinger, Soutschek, Schubert, \& Gerjets, 2017), and increased alpha power may reflect an ease of performance. In addition, faster reaction time resulted in stronger alpha power in frontal and posterior regions during visual memory task (Nenert et al., 2012), which is in line with the current study.

We were unable to replicate the previous study of iTBS demonstrating a significant increase in the accuracy of 2-back task following iTBS compared to sham stimulation (Hoy et al., 2016). The reason for this discrepancy remains unclear, and further research is required as currently only a few studies have been performed in this area to date (Cheng et al., 2016; Debarnot et al., 2015; Demeter, Mirdamadi, Meehan, \& Taylor, 2016; Hoy et al., 2016; Ryals, Rogers, Gross, Polnaszek, $\&$ Voss, 2016). A recent study investigated the effect of prefrontal TBS on a series of cognitive tasks, such as Digits Backward, 3-back task, Stroop Colour and Word Test, and the Tower of Hanoi (Viejo-Sobera et al., 2017). Only subtle behavioural changes were found in these measures in the absence of statistical differences between iTBS, cTBS and sham condition, and with no clear bi-directional changes (i.e., enhanced or impaired performance following iTBS or cTBS, respectively). It is interesting to note that for pre- and post-TBS comparison, iTBS showed improvement in Digits Backward and Stroop WR score.

The absence of strong behavioural changes in the presence of robust neurophysiological effects has also been described following tDCS (Hill et al., 2016), suggesting neurophysiological measures may provide a more sensitive index for assessing changes following neuromodulatory paradigms. It is possible that the marginal behavioural differences between active stimulation conditions may be due to a ceiling effect of performance in healthy individuals. A recent meta-analysis of the working memory performance following noninvasive brain stimulation demonstrates only small effect sizes in improvement in healthy controls compared to clinical populations that showed medium effect sizes (Brunoni \& Vanderhasselt, 2014). Greater behavioural effects may be detected in disorders of WM, such as schizophrenia, in which considerable differences in physiological measures are often observed compared to a control group (Ferrarelli et al., 2012; Noda et al., 2017). However, it is also conceivable that other cognitive tasks may provide more robust behavioural outcome and should further be investigated. The use of TBS as a cognitive enhancer is still at its early stage, and future studies should examine a different variety of cognitive tests in combination with physiological measurement to better characterise the modulatory capacity and the neurobiological basis of TBS on cognition.

\section{4 | Limitations}

Our study design did not include a control site for single-pulse TMS besides the area directly under the iTBS location. Several studies have included the use of a control site such as vertex for TMS (Foltys et al., 2001; Garcia, Grossman, \& Srinivasan, 2011; Silvanto, Cattaneo, Battelli, \& Pascual-Leone, 2008; Taylor, Walsh, \& Eimer, 2008), and this method may provide additional information in future studies. It may be important to note that the spread of neural activity from the vertex stimulation can be observed in brain regions associated with default mode network (DMN) (Jung, Bungert, Bowtell, \& Jackson, 2016), and certain regions of DMN are connected to the working memory network (WMN; DLPFC) in functional connectivity (Piccoli et al., 2015). The highly interconnected nature of prefrontal cortex (Paus, CastroAlamancos, \& Petrides, 2001; Petrides \& Pandya, 2002; Yeterian, Pandya, Tomaiuolo, \& Petrides, 2012) may limit localisation of functionally distant control site. Our TEP data mainly indicate changes in the TEP components which had a high SNR (Supporting Information Table S1). It is possible that increasing the number of stimuli would also have revealed changes in other TEP components. In addition, TMS-EEG at high intensity (120\% rMT) may introduce additional muscle artefacts (Lioumis et al., 2009), which was in part mitigated by stimulating over the F1 electrode to minimise muscle activation (Rogasch et al., 2013b). Muscle artefacts can also be removed effectively via current cleaning method using ICA (Korhonen et al., 2011; Rogasch et al., 2014). Finally, consistency of stimulation site localisation between sessions could be improved by using MRI-guided neuronavigation, however, this was not feasible in the present study. Nevertheless, the TEP waveforms in this study are consistent with previous studies in DLPFC (Chung et al., 
2017; Hill et al., 2017; Rogasch et al., 2014), and comparable results have been reported using EEG-guided methods (Rogasch et al., 2014) and MRI-guided neuronavigation (Lioumis et al., 2009).

\section{5 | CONCLUSION}

The present data provide the first evidence that for iTBS, unlike rTMS (Nahas et al., 2001; Padberg et al., 2002), using higher intensities may not be optimal for maximal neuromodulation, and instead, maximal effects are observed at an intermediate intensity of $75 \%$ rMT. Further research is required to explore whether the present intensity relationship extends to clinical efficacy. The data also indicate that the link between neurophysiological and behavioural effects may not be as direct as hoped, however, it is also possible that repeated sessions are necessary to elicit more robust behavioural outcomes. Despite the modest behavioural outcome in this study, the change in cortical oscillatory activity evoked by TMS has been implicated in clinical improvement in a patient with MDD (a case study) (Pellicciari, Ponzo, Caltagirone, \& Koch, 2017a), and we were able to demonstrate different effects the stimulation intensity has on the oscillatory properties following iTBS over the left prefrontal cortex, which may be useful indices for treatment regime. Other short paradigms of similar duration to TBS are now available (Cash et al., 2016, 2017a), and may offer another option for clinical trials.

In conclusion, the current study indicates that iTBS at 75\% rMT produces the strongest effect on physiological measures in the prefrontal cortex, and increasing the intensity may not necessarily result in a corresponding change. These findings highlight the importance of intensity in administering iTBS and paves the path for more efficacious outcome in patients with neurological and psychiatric disorders.

\section{CONFLICT OF INTEREST}

There are no other conflict.

\section{ORCID}

Sung Wook Chung iD http://orcid.org/0000-0001-8022-8037

\section{REFERENCES}

Aron, A. R. (2007). The neural basis of inhibition in cognitive control. The Neuroscientist, 13, 214-228.

Artola, A., Brocher, S., \& Singer, W. (1990). Different voltage-dependent thresholds for inducing long-term depression and long-term potentiation in slices of rat visual cortex. Nature, 347, 69-72.

Bakker, N., Shahab, S., Giacobbe, P., Blumberger, D. M., Daskalakis, Z. J., Kennedy, S. H., \& Downar, J. (2015). rTMS of the dorsomedial prefrontal cortex for major depression: safety, tolerability, effectiveness, and outcome predictors for $10 \mathrm{~Hz}$ versus intermittent theta-burst stimulation. Brain Stimulation, 8, 208-215.

Batsikadze, G., Moliadze, V., Paulus, W., Kuo, M. F., \& Nitsche, M. A. (2013). Partially non-linear stimulation intensity-dependent effects of direct current stimulation on motor cortex excitability in humans. The Journal of Physiology, 591, 1987-2000.
Benali, A., Trippe, J., Weiler, E., Mix, A., Petrasch-Parwez, E., Girzalsky, W., ... Funke, K. (2011). Theta-burst transcranial magnetic stimulation alters cortical inhibition. Journal of Neuroscience, 31, 1193-1203.

Bonnard, M., Spieser, L., Meziane, H. B., de Graaf, J. B., \& Pailhous, J. (2009). Prior intention can locally tune inhibitory processes in the primary motor cortex: direct evidence from combined TMS-EEG. European Journal of Neuroscience, 30, 913-923.

Bonnefond, M., \& Jensen, O. (2012). Alpha oscillations serve to protect working memory maintenance against anticipated distracters. Current Biology, 22, 1969-1974

Brownjohn, P. W., Reynolds, J. N., Matheson, N., Fox, J., \& Shemmell, J. B. (2014). The effects of individualized theta burst stimulation on the excitability of the human motor system. Brain Stimulation, 7, 260268.

Brunoni, A. R., \& Vanderhasselt, M. A. (2014). Working memory improvement with non-invasive brain stimulation of the dorsolateral prefrontal cortex: a systematic review and meta-analysis. Brain and Cognition, 86, 1-9.

Cardenas-Morales, L., Volz, L. J., Michely, J., Rehme, A. K., Pool, E. M. Nettekoven, C., ... Grefkes, C. (2014). Network connectivity and individual responses to brain stimulation in the human motor system. Cerebral Cortex, 24, 1697-1707.

Cash, R. F., Jegatheeswaran, G., Ni, Z., \& Chen, R. (2017a). Modulation of the direction and magnitude of hebbian plasticity in human motor cortex by stimulus intensity and concurrent inhibition. Brain Stimulation, 10, 83-90.

Cash, R. F., Mastaglia, F. L., \& Thickbroom, G. W. (2013). Evidence for high-fidelity timing-dependent synaptic plasticity of human motor cortex. Journal of Neurophysiology, 109, 106-112.

Cash, R. F., Murakami, T., Chen, R., Thickbroom, G. W., \& Ziemann, U. (2016). Augmenting plasticity induction in human motor cortex by disinhibition stimulation. Cerebral Cortex, 26, 58-69.

Cash, R. F., Noda, Y., Zomorrodi, R., Radhu, N., Farzan, F., Rajji, T. K., ... Blumberger, D. M. (2017b). Characterization of glutamatergic and GABAA-mediated neurotransmission in motor and dorsolateral prefrontal cortex using paired-pulse TMS-EEG. Neuropsychopharmacology, 42, 502-511.

Cash, R. F., Ziemann, U., Murray, K., \& Thickbroom, G. W. (2010). Late cortical disinhibition in human motor cortex: a triple-pulse transcranial magnetic stimulation study. Journal of Neurophysiology, 103, 511518.

Cash, R. F., Ziemann, U., \& Thickbroom, G. W. (2011). Inhibitory and disinhibitory effects on I-wave facilitation in motor cortex. Journal of Neurophysiology, 105, 100-106.

Casula, E. P., Pellicciari, M. C., Picazio, S., Caltagirone, C., \& Koch, G. (2016a). Spike-timing-dependent plasticity in the human dorso-lateral prefrontal cortex. Neuroimage, 143, 204-213.

Casula, E. P., Pellicciari, M. C., Ponzo, V., Stampanoni Bassi, M., Veniero, D., Caltagirone, C., \& Koch, G. (2016b). Cerebellar theta burst stimulation modulates the neural activity of interconnected parietal and motor areas. Scientific Reports, 6, 36191.

Chen, R., Tam, A., Butefisch, C., Corwell, B., Ziemann, U., Rothwell, J. C., \& Cohen, L. G. (1998). Intracortical inhibition and facilitation in different representations of the human motor cortex. Journal of Neurophysiology, 80, 2870-2881.

Chen, Y., \& Huang, X. (2015). Modulation of alpha and beta oscillations during an $\mathrm{n}$-back task with varying temporal memory load. Frontiers in Psychology, 6, 2031.

Cheng, C. M., Juan, C. H., Chen, M. H., Chang, C. F., Lu, H. J., Su, T. P., ... Li, C. T. (2016). Different forms of prefrontal theta burst 
stimulation for executive function of medication- resistant depression: Evidence from a randomized sham-controlled study. Progress in Neuro-Psychopharmacology \& Biological Psychiatry, 66, 35-40.

Chung, S. W., Hoy, K. E., \& Fitzgerald, P. B. (2015a). Theta-burst stimulation: a new form of TMS treatment for depression? Depression and Anxiety, 32, 182-192.

Chung, S. W., Lewis, B. P., Rogasch, N. C., Saeki, T., Thomson, R. H., Hoy, K. E., ... Fitzgerald, P. B. (2017). Demonstration of short-term plasticity in the dorsolateral prefrontal cortex with theta burst stimulation: A TMS-EEG study. Clinical Neurophysiology, 128, 1117-1126.

Chung, S. W., Rogasch, N. C., Hoy, K. E., \& Fitzgerald, P. B. (2015b). Measuring brain stimulation induced changes in cortical properties using TMS-EEG. Brain Stimulation, 8, 1010-1020.

Conforto, A. B., Z'graggen, W. J., Kohl, A. S., Rosler, K. M., \& KaelinLang, A. (2004). Impact of coil position and electrophysiological monitoring on determination of motor thresholds to transcranial magnetic stimulation. Clinical Neurophysiology, 115, 812-819.

Coull, J. T. (1998). Neural correlates of attention and arousal: insights from electrophysiology, functional neuroimaging and psychopharmacology. Progress in Neurobiology, 55, 343-361.

Daskalakis, Z. J., Farzan, F., Barr, M. S., Maller, J. J., Chen, R., \& Fitzgerald, P. B. (2008). Long-interval cortical inhibition from the dorsolateral prefrontal cortex: a TMS-EEG study. Neuropsychopharmacology, 33, 2860-2869.

Davies, C. H., \& Collingridge, G. L. (1996). Regulation of EPSPs by the synaptic activation of GABAB autoreceptors in rat hippocampus. The Journal of Physiology, 496(Pt 2), 451-470.

Davies, C. H., Starkey, S. J., Pozza, M. F., \& Collingridge, G. L. (1991). GABA autoreceptors regulate the induction of LTP. Nature, 349, 609-611.

Debarnot, U., Crepon, B., Orriols, E., Abram, M., Charron, S., Lion, S., ... Piolino, P. (2015). Intermittent theta burst stimulation over left BA10 enhances virtual reality-based prospective memory in healthy aged subjects. Neurobiol Aging, 36, 2360-2369.

Debener, S., \& De Vos, M. (2011). The benefits of simultaneous EEGfMRI for EEG analysis. Clinical Neurophysiology, 122, 217-218.

Deisz, R. A. (1999). The GABA(B) receptor antagonist CGP 55845A reduces presynaptic $G A B A(B)$ actions in neocortical neurons of the rat in vitro. Neuroscience, 93, 1241-1249.

Delorme, A., \& Makeig, S. (2004). EEGLAB: an open source toolbox for analysis of single-trial EEG dynamics including independent component analysis. Journal of Neuroscience Methods, 134, 9-21.

Demeter, E., Mirdamadi, J. L., Meehan, S. K., \& Taylor, S. F. (2016). Short theta burst stimulation to left frontal cortex prior to encoding enhances subsequent recognition memory. Cognitive, Affective, \& Behavioral Neuroscience, 16, 724-735.

Desmyter, S., Duprat, R., Baeken, C., Van Autreve, S., Audenaert, K., \& van Heeringen, K. (2016). Accelerated intermittent theta burst stimulation for suicide risk in therapy-resistant depressed patients: A randomized, sham-controlled trial. Frontiers in Human Neuroscience, 10, 480.

Di Lazzaro, V., Dileone, M., Pilato, F., Capone, F., Musumeci, G., Ranieri, F., ... Profice, P. (2011). Modulation of motor cortex neuronal networks by rTMS: comparison of local and remote effects of six different protocols of stimulation. Journal of Neurophysiology, 105, 2150-2156.

Doeltgen, S. H., \& Ridding, M. C. (2011). Low-intensity, short-interval theta burst stimulation modulates excitatory but not inhibitory motor networks. Clinical Neurophysiology, 122, 1411-1416.

Dosenbach, N. U., Fair, D. A., Cohen, A. L., Schlaggar, B. L., \& Petersen, S. E. (2008). A dual-networks architecture of top-down control. Trends in Cognitive Sciences, 12, 99-105.
Duprat, R., Desmyter, S., Rudi de, R., van Heeringen, K., Van den Abbeele, D., Tandt, H., ... Baeken, C. (2016). Accelerated intermittent theta burst stimulation treatment in medication-resistant major depression: A fast road to remission? Journal of Affective Disorders, 200, 6-14.

Farzan, F., Barr, M. S., Hoppenbrouwers, S. S., Fitzgerald, P. B., Chen, R., Pascual-Leone, A., \& Daskalakis, Z. J. (2013). The EEG correlates of the TMS-induced EMG silent period in humans. Neuroimage, 83 120-134.

Farzan, F., Barr, M. S., Levinson, A. J., Chen, R., Wong, W., Fitzgerald, P. B., \& Daskalakis, Z. J. (2010). Reliability of long-interval cortical inhibition in healthy human subjects: a TMS-EEG study. Journal of Neurophysiology, 104, 1339-1346.

Farzan, F., Barr, M. S., Wong, W., Chen, R., Fitzgerald, P. B., \& Daskalakis, Z. J. (2009). Suppression of gamma-oscillations in the dorsolateral prefrontal cortex following long interval cortical inhibition: a TMSEEG study. Neuropsychopharmacology, 34, 1543-1551.

Farzan, F., Vernet, M., Shafi, M. M., Rotenberg, A., Daskalakis, Z. J., \& Pascual-Leone, A. (2016). Characterizing and modulating brain circuitry through transcranial magnetic stimulation combined with electroencephalography. Frontiers in Neural Circuits, 10, 73.

Ferrarelli, F., Sarasso, S., Guller, Y., Riedner, B. A., Peterson, M. J., Bellesi, M., ... Tononi, G. (2012). Reduced natural oscillatory frequency of frontal thalamocortical circuits in schizophrenia. Archives of General Psychiatry, 69, 766-774.

Folstein, J. R., \& Van Petten, C. (2008). Influence of cognitive control and mismatch on the N2 component of the ERP: a review. Psychophysiology, 45, 152-170.

Foltys, H., Sparing, R., Boroojerdi, B., Krings, T., Meister, I. G., Mottaghy, F. M., \& Topper, R. (2001). Motor control in simple bimanual movements: a transcranial magnetic stimulation and reaction time study. Clinical Neurophysiology, 112, 265-274.

Garcia, J. O., Grossman, E. D., \& Srinivasan, R. (2011). Evoked potentials in large-scale cortical networks elicited by TMS of the visual cortex. Journal of Neurophysiology, 106, 1734-1746.

Gentner, R., Wankerl, K., Reinsberger, C., Zeller, D., \& Classen, J. (2008). Depression of human corticospinal excitability induced by magnetic theta-burst stimulation: evidence of rapid polarity-reversing metaplasticity. Cerebral Cortex, 18, 2046-2053.

George, M. S., Lisanby, S. H., Avery, D., McDonald, W. M., Durkalski, V., Pavlicova, M., ... Sackeim, H. A. (2010). Daily left prefrontal transcranial magnetic stimulation therapy for major depressive disorder: a sham-controlled randomized trial. Archives of General Psychiatry, 67, 507-516.

George, M. S., Taylor, J. J., \& Short, E. B. (2013). The expanding evidence base for rTMS treatment of depression. Current Opinion in Psychiatry, 26, 13-18.

Goldsworthy, M. R., Pitcher, J. B., \& Ridding, M. C. (2012). The application of spaced theta burst protocols induces long-lasting neuroplastic changes in the human motor cortex. European Journal of Neuroscience, 35, 125-134.

Goldsworthy, M. R., Pitcher, J. B., \& Ridding, M. C. (2013). Neuroplastic modulation of inhibitory motor cortical networks by spaced theta burst stimulation protocols. Brain Stimulation, 6, 340-345.

Gramfort, A., Papadopoulo, T., Olivi, E., \& Clerc, M. (2010). OpenMEEG: opensource software for quasistatic bioelectromagnetics. Biomedical Engineering Online, 9, 45.

Haatveit, B. C., Sundet, K., Hugdahl, K., Ueland, T., Melle, I., \& Andreassen, O. A. (2010). The validity of $d$ prime as a working memory index: results from the "Bergen n-back task. Journal of Clinical and Experimental Neuropsychology, 32, 871-880. 
Hameed, M. Q., Dhamne, S. C., Gersner, R., Kaye, H. L., Oberman, L. M., Pascual-Leone, A., \& Rotenberg, A. (2017). Transcranial magnetic and direct current stimulation in children. Current Neurology and Neuroscience Reports, 17, 11.

Harrington, A., \& Hammond-Tooke, G. D. (2015). Theta burst stimulation of the cerebellum modifies the TMS-Evoked N100 potential, a marker of GABA inhibition. PLoS One, 10, e0141284.

Hedges, L. V., \& Olkin, I. (1985). Statistical methods for meta-analysis. Orlando, FL: Academic Press.

Hill, A. T., Rogasch, N. C., Fitzgerald, P. B., \& Hoy, K. E. (2016). TMSEEG: $A$ window into the neurophysiological effects of transcranial electrical stimulation in non-motor brain regions. Neuroscience \& Biobehavioral Reviews, 64, 175-184.

Hill, A. T., Rogasch, N. C., Fitzgerald, P. B., \& Hoy, K. E. (2017). Effects of prefrontal bipolar and high-definition transcranial direct current stimulation on cortical reactivity and working memory in healthy adults. Neuroimage, 152, 142-157.

Howard, M. W., Rizzuto, D. S., Caplan, J. B., Madsen, J. R., Lisman, J., Aschenbrenner-Scheibe, R., ... Kahana, M. J. (2003). Gamma oscillations correlate with working memory load in humans. Cerebral Cortex, 13, 1369-1374.

Hoy, K. E., Bailey, N., Michael, M., Fitzgibbon, B. M., Rogasch, N. C., Saeki, T., \& Fitzgerald, P. B. (2016). Enhancement of working memory and task-related oscillatory activity following intermittent theta burst stimulation in healthy controls. Cerebral Cortex, 26, 4563-4573.

Hsieh, L. T., \& Ranganath, C. (2014). Frontal midline theta oscillations during working memory maintenance and episodic encoding and retrieval. Neuroimage, 85 Pt 2, 721-729.

Hu, L., Mouraux, A., Hu, Y., \& lannetti, G. D. (2010). A novel approach for enhancing the signal-to-noise ratio and detecting automatically event-related potentials (ERPs) in single trials. Neuroimage, 50, 99111.

Huang, G., \& Mouraux, A. (2015). MEP latencies predict the neuromodulatory effect of cTBS delivered to the ipsilateral and contralateral sensorimotor cortex. PLoS One, 10, e0133893.

Huang, Y. Z., Edwards, M. J., Rounis, E., Bhatia, K. P., \& Rothwell, J. C. (2005). Theta burst stimulation of the human motor cortex. Neuron, 45, 201-206.

IImoniemi, R. J., \& Kicic, D. (2010). Methodology for combined TMS and EEG. Brain Topography , 22, 233-248.

Jones, C. B., Lulic, T., Bailey, A. Z., Mackenzie, T. N., Mi, Y. Q., Tommerdahl, M., \& Nelson, A. J. (2016). Metaplasticity in human primary somatosensory cortex: effects on physiology and tactile perception. Journal of Neurophysiology, 115, 2681-2691.

Jung, J., Bungert, A., Bowtell, R., \& Jackson, S. R. (2016). Vertex stimulation as a control site for transcranial magnetic stimulation: A concurrent TMS/fMRI study. Brain Stimulation, 9, 58-64.

Kahkonen, S., Komssi, S., Wilenius, J., \& Ilmoniemi, R. J. (2005). Prefrontal TMS produces smaller EEG responses than motor-cortex TMS: implications for rTMS treatment in depression. Psychopharmacology (Berl), 181, 16-20.

Kahkonen, S., Wilenius, J., Nikulin, V. V., Ollikainen, M., \& Ilmoniemi, R. J. (2003). Alcohol reduces prefrontal cortical excitability in humans: a combined TMS and EEG study. Neuropsychopharmacology, 28, 747-754.

Koessler, L., Maillard, L., Benhadid, A., Vignal, J. P., Felblinger, J., Vespignani, H., \& Braun, M. (2009). Automated cortical projection of EEG sensors: anatomical correlation via the international $10-10$ system. Neuroimage, 46, 64-72.

Kok, A. (2001). On the utility of P3 amplitude as a measure of processing capacity. Psychophysiology, 38, 557-577.
Kopp, B., Rist, F., \& Mattler, U. (1996). N200 in the flanker task as a neurobehavioral tool for investigating executive control. Psychophysiology, 33, 282-294.

Korhonen, R. J., Hernandez-Pavon, J. C., Metsomaa, J., Maki, H., Ilmoniemi, R. J., \& Sarvas, J. (2011). Removal of large muscle artifacts from transcranial magnetic stimulation-evoked EEG by independent component analysis. Medical \& Biological Engineering \& Computing, 49, 397-407.

Kumpulainen, S., Mrachacz-Kersting, N., Peltonen, J., Voigt, M., \& Avela, J. (2012). The optimal interstimulus interval and repeatability of paired associative stimulation when the soleus muscle is targeted. Experimental Brain Research, 221, 241-249.

Larson, J., \& Lynch, G. (1986). Induction of synaptic potentiation in hippocampus by patterned stimulation involves two events. Science, 232, 985-988

Larson, J., \& Munkacsy, E. (2015). Theta-burst LTP. Brain Research, 1621 , 38-50.

Larson, J., Wong, D., \& Lynch, G. (1986). Patterned stimulation at the theta frequency is optimal for the induction of hippocampal longterm potentiation. Brain Research, 368, 347-350.

Li, C. T., Chen, M. H., Juan, C. H., Huang, H. H., Chen, L. F., Hsieh, J. C., ... Su, T. P. (2014). Efficacy of prefrontal theta-burst stimulation in refractory depression: a randomized sham-controlled study. Brain, 137, 2088-2098.

Lioumis, P., Kicic, D., Savolainen, P., Makela, J. P., \& Kahkonen, S. (2009). Reproducibility of TMS-Evoked EEG responses. Human Brain Mapping, 30, 1387-1396.

Lisman, J. (2010). Working memory: the importance of theta and gamma oscillations. Current Biology, 20, R490-R492.

Lisman, J. E., \& Jensen, O. (2013). The theta-gamma neural code. Neuron, 77, 1002-1016.

Luscher, C., \& Malenka, R. C. (2012). NMDA receptor-dependent longterm potentiation and long-term depression (LTP/LTD). Cold Spring Harbor Perspectives in Biology, 4 ,

Machado, S., Arias-Carrion, O., Paes, F., Vieira, R. T., Caixeta, L., Novaes, F., ... Nardi, A. E. (2013). Repetitive transcranial magnetic stimulation for clinical applications in neurological and psychiatric disorders: an overview. The Eurasian Journal of Medicine, 45, 191-206.

Manza, P., Hau, C. L., \& Leung, H. C. (2014). Alpha power gates relevant information during working memory updating. Journal of Neuroscience, 34, 5998-6002.

Moliadze, V., Atalay, D., Antal, A., \& Paulus, W. (2012). Close to threshold transcranial electrical stimulation preferentially activates inhibitory networks before switching to excitation with higher intensities. Brain Stimulation, 5, 505-511.

Moore, R. A., Gale, A., Morris, P. H., \& Forrester, D. (2008). Alpha power and coherence primarily reflect neural activity related to stages of motor response during a continuous monitoring task. International Journal of Psychophysiology, 69, 79-89.

Mott, D. D., \& Lewis, D. V. (1991). Facilitation of the induction of longterm potentiation by GABAB receptors. Science, 252, 1718-1720.

Nahas, Z., Lomarev, M., Roberts, D. R., Shastri, A., Lorberbaum, J. P., Teneback, C., ... Bohning, D. E. (2001). Unilateral left prefrontal transcranial magnetic stimulation (TMS) produces intensitydependent bilateral effects as measured by interleaved BOLD fMRI. Biological Psychiatry, 50, 712-720.

Nenert, R., Viswanathan, S., Dubuc, D. M., \& Visscher, K. M. (2012). Modulations of ongoing alpha oscillations predict successful shortterm visual memory encoding. Frontiers in Human Neuroscience, 6, 127. 
Nettekoven, C., Volz, L. J., Kutscha, M., Pool, E. M., Rehme, A. K., Eickhoff, S. B., ... Grefkes, C. (2014). Dose-dependent effects of theta burst rTMS on cortical excitability and resting-state connectivity of the human motor system. Journal of Neuroscience, 34, 6849-6859.

Nikulin, V. V., Kicic, D., Kahkonen, S., \& Ilmoniemi, R. J. (2003). Modulation of electroencephalographic responses to transcranial magnetic stimulation: evidence for changes in cortical excitability related to movement. European Journal of Neuroscience, 18, 1206-1212.

Noda, Y., Barr, M.S., Zomorrodi, R., Cash, R. F., Rajji, T. K., Farzan, F., ... Blumberger, D. M. (2017). Reduced short-latency afferent inhibition in prefrontal but not motor cortex and its association with executive function in schizophrenia: a combined TMS-EEG study. Schizophrenia Bulletin, sbx041.

Noda, Y., Cash, R. F., Zomorrodi, R., Dominguez, L. G., Farzan, F., Rajji, T. K., ... Blumberger, D. M. (2016). A combined TMS-EEG study of short-latency afferent inhibition in the motor and dorsolateral prefrontal cortex. Journal of Neurophysiology, 116, 938-948.

O’Reardon, J. P., Cristancho, P., Pilania, P., Bapatla, K. B., Chuai, S., \& Peshek, A. D. (2007). Patients with a major depressive episode responding to treatment with repetitive transcranial magnetic stimulation (rTMS) are resistant to the effects of rapid tryptophan depletion. Depression and Anxiety, 24, 537-544.

Oostenveld, R., Fries, P., Maris, E., \& Schoffelen, J. M. (2011). FieldTrip: Open source software for advanced analysis of MEG, EEG, and invasive electrophysiological data. Computational Intelligence and Neuroscience, 2011, 156869.

Otis, T. S., De Koninck, Y., \& Mody, I. (1993). Characterization of synaptically elicited GABAB responses using patch-clamp recordings in rat hippocampal slices. The Journal of Physiology, 463, 391-407.

Pacelli, G. J., Su, W., \& Kelso, S. R. (1989). Activity-induced depression of synaptic inhibition during LTP-inducing patterned stimulation. Brain Research, 486, 26-32.

Padberg, F., Zwanzger, P., Keck, M. E., Kathmann, N., Mikhaiel, P., Ella, R., ... Moller, H. J. (2002). Repetitive transcranial magnetic stimulation (rTMS) in major depression: relation between efficacy and stimulation intensity. Neuropsychopharmacology, 27, 638-645.

Paus, T., Castro-Alamancos, M. A., \& Petrides, M. (2001). Cortico-cortical connectivity of the human mid-dorsolateral frontal cortex and its modulation by repetitive transcranial magnetic stimulation. European Journal of Neuroscience, 14, 1405-1411.

Pedapati, E. V., Gilbert, D. L., Erickson, C. A., Horn, P. S., Shaffer, R. C., Wink, L. K., ... Wu, S. W. (2016). Abnormal cortical plasticity in youth with autism spectrum disorder: A transcranial magnetic stimulation case-control pilot study. Journal of Child and Adolescent Psychopharmacology, 26, 625-631.

Pedapati, E. V., Gilbert, D. L., Horn, P. S., Huddleston, D. A., Laue, C. S., Shahana, N., \& Wu, S. W. (2015). Effect of $30 \mathrm{~Hz}$ theta burst transcranial magnetic stimulation on the primary motor cortex in children and adolescents. Frontiers in Human Neuroscience, 9, 91.

Pellicciari, M. C., Ponzo, V., Caltagirone, C., \& Koch, G. (2017a). Restored asymmetry of prefrontal cortical oscillatory activity after bilateral theta burst stimulation treatment in a patient with major depressive disorder: A TMS-EEG study. Brain Stimulation, 10, 147-149.

Pellicciari, M. C., Veniero, D., \& Miniussi, C. (2017b). Characterizing the cortical oscillatory response to TMS pulse. Frontiers in Cellular Neuroscience, 11, 38.

Petrides, M., \& Pandya, D. N. (2002). Comparative cytoarchitectonic analysis of the human and the macaque ventrolateral prefrontal cortex and corticocortical connection patterns in the monkey. European Journal of Neuroscience, 16, 291-310.

Piccoli, T., Valente, G., Linden, D. E., Re, M., Esposito, F., Sack, A. T., \& Di Salle, F. (2015). The default mode network and the working memory network are not anti-correlated during all phases of a working memory task. PLoS One, 10, e0123354.

Plewnia, C., Pasqualetti, P., Grosse, S., Schlipf, S., Wasserka, B., Zwissler, B., \& Fallgatter, A. (2014). Treatment of major depression with bilateral theta burst stimulation: a randomized controlled pilot trial. Journal of Affective Disorders, 156, 219-223.

Prasser, J., Schecklmann, M., Poeppl, T. B., Frank, E., Kreuzer, P. M., Hajak, G., ... Langguth, B. (2015). Bilateral prefrontal rTMS and theta burst TMS as an add-on treatment for depression: a randomized placebo controlled trial. World Journal of Biological Psychiatry, 16, 57-65.

Premoli, I., Rivolta, D., Espenhahn, S., Castellanos, N., Belardinelli, P., Ziemann, U., \& Muller-Dahlhaus, F. (2014). Characterization of GABABreceptor mediated neurotransmission in the human cortex by pairedpulse TMS-EEG. Neuroimage, 103, 152-162.

Rogasch, N. C., Daskalakis, Z. J., \& Fitzgerald, P. B. (2013a). Mechanisms underlying long-interval cortical inhibition in the human motor cortex: a TMS-EEG study. Journal of Neurophysiology, 109, 89-98.

Rogasch, N. C., Daskalakis, Z. J., \& Fitzgerald, P. B. (2015). Cortical inhibition of distinct mechanisms in the dorsolateral prefrontal cortex is related to working memory performance: a TMS-EEG study. Cortex, $64,68-77$.

Rogasch, N. C., Sullivan, C., Thomson, R. H., Rose, N. S., Bailey, N. W., Fitzgerald, P. B., ... Hernandez-Pavon, J. C. (2017). Analysing concurrent transcranial magnetic stimulation and electroencephalographic data: a review and introduction to the open-source TESA software. Neuroimage.

Rogasch, N. C., Thomson, R. H., Daskalakis, Z. J., \& Fitzgerald, P. B. (2013b). Short-latency artifacts associated with concurrent TMS-EEG. Brain Stimulation, 6, 868-876.

Rogasch, N. C., Thomson, R. H., Farzan, F., Fitzgibbon, B. M., Bailey, N. W., Hernandez-Pavon, J. C., ... Fitzgerald, P. B. (2014). Removing artefacts from TMS-EEG recordings using independent component analysis: importance for assessing prefrontal and motor cortex network properties. Neuroimage, 101, 425-439.

Rousselet, G. A. (2012). Does filtering preclude us from studying ERP time-courses? Frontiers in Psychology, 3, 131.

Ryals, A. J., Rogers, L. M., Gross, E. Z., Polnaszek, K. L., \& Voss, J. L. (2016). Associative recognition memory awareness improved by theta-burst stimulation of frontopolar cortex. Cerebral Cortex, 26, 1200-1210.

Sasaki, K., Gemba, H., \& Tsujimoto, T. (1989). Suppression of visually initiated hand movement by stimulation of the prefrontal cortex in the monkey. Brain Research, 495, 100-107.

Scharinger, C., Soutschek, A., Schubert, T., \& Gerjets, P. (2017). Comparison of the working memory load in N-Back and working memory span tasks by means of EEG frequency band power and P300 amplitude. Frontiers in Human Neuroscience, 11, 6.

Schmajuk, M., Liotti, M., Busse, L., \& Woldorff, M. G. (2006). Electrophysiological activity underlying inhibitory control processes in normal adults. Neuropsychologia, 44, 384-395.

Sheehan, D. V., Lecrubier, Y., Sheehan, K. H., Amorim, P., Janavs, J., Weiller, E., ... Dunbar, G. C. (1998). The Mini-International Neuropsychiatric Interview (M.I.N.I.): The development and validation of a structured diagnostic psychiatric interview for DSM-IV and ICD-10. Journal of Clinical Psychiatry, 59(Suppl 20), 22-33. quiz 34-57.

Silvanto, J., Cattaneo, Z., Battelli, L., \& Pascual-Leone, A. (2008). Baseline cortical excitability determines whether TMS disrupts or facilitates behavior. Journal of Neurophysiology, 99, 2725-2730.

Suppa, A., Ortu, E., Zafar, N., Deriu, F., Paulus, W., Berardelli, A., \& Rothwell, J. C. (2008). Theta burst stimulation induces after-effects on 
contralateral primary motor cortex excitability in humans. The Journal of Physiology, 586, 4489-4500.

Tadel, F., Baillet, S., Mosher, J. C., Pantazis, D., \& Leahy, R. M. (2011). Brainstorm: a user-friendly application for MEG/EEG analysis. Computational Intelligence and Neuroscience, 2011, 879716.

Taylor, P. C., Walsh, V., \& Eimer, M. (2008). Combining TMS and EEG to study cognitive function and cortico-cortico interactions. Behavioural Brain Research, 191, 141-147.

Thomson, R. H., Cleve, T. J., Bailey, N. W., Rogasch, N. C., Maller, J. J., Daskalakis, Z. J., \& Fitzgerald, P. B. (2013). Blood oxygenation changes modulated by coil orientation during prefrontal transcranial magnetic stimulation. Brain Stimulation, 6, 576-581.

Tsang, P., Jacobs, M. F., Lee, K. G., Asmussen, M. J., Zapallow, C. M., \& Nelson, A. J. (2014). Continuous theta-burst stimulation over primary somatosensory cortex modulates short-latency afferent inhibition. Clinical Neurophysiology, 125, 2253-2259.

Turrigiano, G. G., \& Nelson, S. B. (2004). Homeostatic plasticity in the developing nervous system. Nature Reviews Neuroscience, 5, 97107.

van Ede, F., \& Maris, E. (2016). Physiological plausibility can increase reproducibility in cognitive neuroscience. Trends in Cognitive Sciences, 20, 567-569.

Vernet, M., Bashir, S., Yoo, W. K., Perez, J. M., Najib, U., \& PascualLeone, A. (2013). Insights on the neural basis of motor plasticity induced by theta burst stimulation from TMS-EEG. European Journal of Neuroscience, 37, 598-606.

Viejo-Sobera, R., Redolar-Ripoll, D., Boixados, M., Palaus, M., ValeroCabre, A., \& Marron, E. M. (2017). Impact of Prefrontal Theta Burst Stimulation on Clinical Neuropsychological Tasks. Frontiers in Neuroscience, 11, 462.

Vogel, E. K., \& Luck, S. J. (2000). The visual N1 component as an index of a discrimination process. Psychophysiology, 37, 190-203.
Vossen, A., Gross, J., \& Thut, G. (2015). Alpha power increase after transcranial alternating current stimulation at alpha frequency (alphatACS) reflects plastic changes rather than entrainment. Brain Stimulation, 8, 499-508.

Wozniak-Kwasniewska, A., Szekely, D., Aussedat, P., Bougerol, T., \& David, O. (2014). Changes of oscillatory brain activity induced by repetitive transcranial magnetic stimulation of the left dorsolateral prefrontal cortex in healthy subjects. Neuroimage, 88, 91-99.

Xu, G., Lan, Y., Huang, D., Chen, S., Chen, L., Zeng, J., \& Pei, Z. (2013). The study on the frontoparietal networks by continuous theta burst stimulation in healthy human subjects. Behavioural Brain Research, 240, 60-68.

Yeterian, E. H., Pandya, D. N., Tomaiuolo, F., \& Petrides, M. (2012). The cortical connectivity of the prefrontal cortex in the monkey brain. Cortex, 48, 58-81.

Zheng, C., \& Zhang, T. (2015). Synaptic plasticity-related neural oscillations on hippocampus-prefrontal cortex pathway in depression. Neuroscience, 292, 170-180.

\section{SUPPORTING INFORMATION}

Additional Supporting Information may be found online in the supporting information tab for this article.

How to cite this article: Chung SW, Rogasch NC, Hoy KE, Sullivan CM, Cash RFH, Fitzgerald PB. Impact of different intensities of intermittent theta burst stimulation on the cortical properties during TMS-EEG and working memory performance. Hum Brain Mapp. 2018;39:783-802. https://doi.org/10.1002/hbm.23882 